\title{
Trapping and Cascading of Eigenvalues In the Large Coupling Limit
}

\author{
F. Gesztesy ${ }^{1,4, \star \star \star \star}$, D. Gurarie ${ }^{1, \star \star \star}$, H. Holden ${ }^{2}$, M. Klaus ${ }^{3}$, L. Sadun ${ }^{1}$, \\ B. Simon ${ }^{1, \star \star}$, and P. Vogl ${ }^{4}$ \\ ${ }^{1}$ Division of Physics, Mathematics, and Astronomy, California Institute of Technology, Pasadena, \\ CA 91125, USA \\ ${ }^{2}$ Institute of Mathematics, University of Trondheim, N-7034 Trondheim-NTH, Norway \\ ${ }^{3}$ Department of Mathematics, Virginia Polytechnic Institute and State University, Blacksburg, VA \\ 24061, USA \\ ${ }^{4}$ Institute for Theoretical Physics, University of Graz, A-8010 Graz, Austria
}

\begin{abstract}
We consider eigenvalues $E_{\lambda}$ of the Hamiltonian $H_{\lambda}=-\Delta+V+$ $\lambda W, W$ compactly supported, in the $\lambda \rightarrow \infty$ limit. For $W \geqq 0$ we find monotonic convergence of $E_{\lambda}$ to the eigenvalues of a limiting operator $H_{\infty}$ (associated with an exterior Dirichlet problem), and we estimate the rate of convergence for 1-dimensional systems. In 1-dimensional systems with $W \leqq 0$, or with $W$ changing sign, we do not find convergence. Instead, we find a cascade phenomenon, in which, as $\lambda \rightarrow \infty$, each eigenvalue $E_{\lambda}$ stays near a Dirichlet eigenvalue for a long interval (of length $O(\sqrt{\lambda})$ ) of the scaling range, quickly drops to the next lower Dirichlet eigenvalue, stays there for a long interval, drops again, and so on. As a result, for most large values of $\lambda$ the discrete spectrum of $H_{\lambda}$ is close to that of $H_{\infty}$, but when $\lambda$ reaches a transition region, the entire spectrum quickly shifts down by one. We also explore the behavior of several explicit models, as $\lambda \rightarrow \infty$.
\end{abstract}

\section{Introduction}

In quantum mechanics one frequently encounters Hamiltonians of the form $H_{\lambda}=H_{0}+\lambda W$, where $H_{0}$ describes a well-understood system (the "background" or "free" Hamiltonian), $W$ describes any of various interactions in the system (e.g. interacting particles, and external fields, etc.), and $\lambda$ (the "coupling constant") measures the strength of the interaction $W$. In this paper we consider Hamiltonians

\footnotetext{
$\star$ Max Kade Foundation Fellow

$\star \star$ Partially supported by USNSF under Grant DMS-8416049

$\star \star \star$ On leave of absence from Department of Mathematics and Statistics, Case Western Reserve University, Cleveland, OH 44106, USA. Partially supported by USNSF under Grant DMS-8620231 and the Case Institute of Technology, RIG
} 
$H_{0}=-\Delta+V(x)$ on $\mathbf{R}^{v}$ and compactly supported perturbations $W(x)$, in the $\lambda \rightarrow \infty$ limit. In particular we consider the behavior of specific eigenvalues $E_{\lambda}$ of $H_{\lambda}$ as a function of $\lambda$ for large $\lambda$.

Large-coupling problems (sometimes called "singular perturbations"), have appeared in a variety of context. We give some examples.

Harrell [12] studied the large-coupling limit of the Hill operator $H_{\lambda}=$ $-\Delta+\lambda W(x)$, with periodic $W$ on $\mathbf{R}$. (This is, strictly speaking, not an eigenvalue problem at all, as $H_{\lambda}$ has a continuous spectrum made of bands and gaps.) $\mathrm{He}$ showed that, as $\lambda \rightarrow \infty$, all the bands shrink at an exponential rate to certain limiting expressions, which coincide with eigenvalues of a harmonic oscillator.

Another class of operators, studied by Ashbaugh and Harrell [4], are Schrödinger operators of the form $H_{\lambda}=-\Delta+V+\lambda W$ on $\mathbf{R}^{v}$, where the support of $W$ is restricted to the exterior of a bounded region in $\mathbf{R}^{v}$. It was shown (under certain regularity assumptions on the behavior of $W$ at $\infty$ ) that each eigenvalue $E_{\lambda}$ of $H_{\lambda}$ converges to a limiting value $E_{0}$, an eigenvalue of the interior Dirichlet problem. Moreover, $E_{\lambda}$ was shown to admit a Puiseux-type expansion $E_{\lambda}=\sum_{n} a_{n} \lambda^{-r n}$ in certain negative fractional powers of $\lambda$.

Operators of the form $H_{0}+\lambda W, W$ compactly supported, have appeared as mathematical models of semiconductors with impurities [23,33]. The background Hamiltonian here is a Hill (Bloch) operator $H_{0}=-\Delta+V$ on $\mathbf{R}^{v}$ or $\mathbf{Z}^{v}$ with a periodic or quasiperiodic potential $V$, reflecting the periodic or quasiperiodic arrangement of atoms in a crystalline lattice. $W$ represents the effect of an localized impurity. $\lambda$ measures the "depth of impurity levels" (see [33]).

In this last case, the spectrum of the Hill operator $H_{0}$ is well known to exhibit a band and gap structure, characterized by continuous energy intervals separated by gaps. Adding a perturbation $\lambda W$ typically results in eigenvalues $E_{\lambda}$ appearing in the gaps, and one is interested in the behavior of $E_{\lambda}$ as $\lambda$ varies in $\mathbf{R}$. One interesting question is whether the range of $E_{\lambda}$ covers the spectral gaps of $H_{0}$. This question was recently addressed by Deift and Hempel (see $[2,8,10,13,20]$ ).

In many of these examples of large coupling, it was demonstrated that each $E_{\lambda}$ converges as $\lambda \rightarrow \infty$ to a limiting value $E_{\infty}$, and that the limiting values ("pinning" or "trapping" levels) are eigenvalues of a limiting operator $H_{\infty}$. In the case of interest to us, namely localized perturbations $\lambda W$ of Schrödinger operators $-\Delta+V$ on $\mathbf{R}^{v}$, the natural candidate for $H_{\infty}$ is $-\Delta+V$ restricted to $\mathbf{R}^{v} \backslash \Sigma$ ( $\Sigma$ denoting the support of $W$ ) with a Dirichlet condition on the boundary of $\Sigma$. We call the set of eigenvalues of this Dirichlet operator $\left\{E_{\infty}\right\}$.

In this paper, we ask whether each $E_{\lambda}$ converges to an $E_{\infty}$ as $\lambda \rightarrow \infty$, and if so at what rate?

In Sect. 2 we show that, for repulsive potentials $W \geqq 0$, the eigenvalues of $H_{\lambda}$ do converge to those of $H_{\infty}$, as a consequence of the monotonic convergence in norm of the resolvent $\left(H_{\lambda}-z\right)^{-1}$ to the pseudoresolvent $\left(H_{\infty}-z\right)^{-1} \oplus 0$. In Sect. 3 we narrow our scope to 1-dimensional problems, and calculate the rate at which $E_{\lambda} \uparrow E_{\infty}$.

In Sect. 4 we see that, for $W$ attractive or of mixed sign, resolvent convergence fails in any meaningful sense. Instead we see a new and intriguing phenomenon 


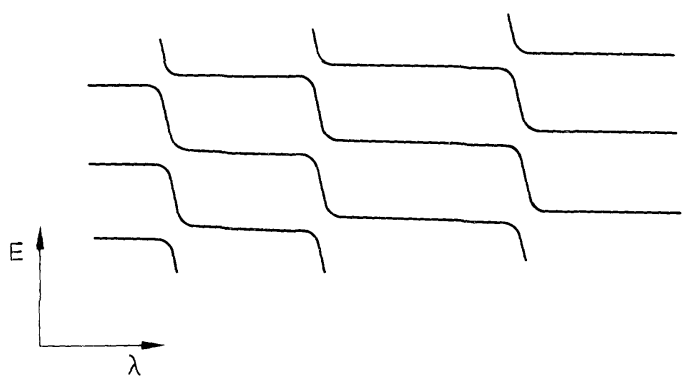

Fig. 1.1. The cascade phenomenon

of cascading eigenvalues. This cascade phenomenon, shown in Fig. 1.1, is defined precisely in Sect. 5, but roughly it means that each eigenvalue $E_{\lambda}$ as a function of $\lambda$ spends most of the scaling range (increasing intervals of length $O(\sqrt{\lambda})$ ) in the vicinity of Dirichlet levels $\left\{E_{\infty}\right\}$. These almost flat regions (plateaus) on the curve $E=E_{\lambda}$ are followed by relatively short intervals of rapid transition, where $E_{\lambda}$ drops down from one Dirichlet level to the next lower one. The pattern repeats itself (and becomes asymptotically more pronounced as $\lambda \rightarrow \infty$ ) until $E_{\lambda}$ hits the lowest (ground state) Dirichlet level, or reaches the bottom of a spectral gap, after which it goes to $-\infty$ or disappears. This phenomenon may be roughly understood as a partial decoupling of the interior and exterior problems, together with the no-crossing rule for eigenvalue trajectories.

It should be noted that a special case of the cascade phenomenon was first observed by Zel'dovich [36] in a different context (energy levels in a distorted Coulomb potential), and is known in the physics literature as the Zel'dovich phenomenon $[11,21,24]$.

For semiconductors with deep level impurities, the cascade phenomenon could provide a possible mechanism for experimentally observed pinning of energy eigenvalue levels as $\lambda \rightarrow \infty[23,33]$, and would identify these levels as exterior Dirichlet eigenvalues. Although the eigenvalues $E_{\lambda}$ do not in fact converge to anything, the large size of the plateaus compared to the transition regions indicates that experiments at large $\lambda$ are likely to find eigenvalues near all the Dirichlet levels, and nowhere else.

In Sect. 5 we study in detail various features of the cascade phenomenon in attractive potentials $W$. This analysis includes the size and location of the plateaus and transition regions, approximate shapes of the eigenvalue trajectories, and separations at near-crossings of neighboring eigenvalue trajectories. In Sect. 6 we extend this analysis to the entirely new case of potentials $W$ that change sign.

After the detailed study of Schrödinger operators with localized perturbations in Sects. 2-6, we turn to some other examples and models. In Sect. 7 we consider a perturbation of point support, $W= \pm \delta(x)$, on the periodic background of the diatomic Kronig-Penney model [27]. In this example the cascade phenomenon disappears, and we get norm-resolvent convergence of $H_{\lambda}$ to a limiting operator, for both cases $W= \pm \delta(x)$. This is consistent with the results of Sects. 5-6, in which 
we see that all essential features of the cascade phenomenon (rates of convergence, location of the transition regions, etc.) depend on a certain integral that vanishes as $W \rightarrow \pm \delta(x)$. The dependence is such that, as the integral goes to zero, all transitions move to $\infty$, so the first plateau stretches over the entire range $[0, \infty)$ of the scaling parameter $\lambda$.

In Sect. 8 we consider Dirac operators. Under certain circumstances eigenvalue trapping occurs, but the cascade phenomenon is never seen.

The different parts of this paper use very different methods to treat somewhat different cases. Sections 2 and 4 treat resolvent convergence in $v$ dimensions utilizing mostly functional analytic tools. Sections 3,5, and 6 treat 1 -dimensional systems, with $H_{0}=-d^{2} / d x^{2}+V$ and $\operatorname{supp}(W)=[0, l]$, and exploit WKB machinery. (The general $v$-dimensional case of attractive and mixed perturbations remains open, largely due to technical difficulties involved in a multidimensional WKB approach). Sections 7 and 8 deal with specific models and involve explicit calculations.

We tried to organize this paper so that these various parts could, in principle, be read independently.

\section{The Repulsive Case in $v$ Dimensions}

In this section we consider the case $W \geqq 0, \lambda \rightarrow \infty$. Our main hypotheses read: (H.2.1) $V \in L^{\infty}\left(\mathbf{R}^{v}\right)$ real-valued, $v \in \mathbf{N}$.

(H.2.2) $0 \leqq W \in L^{\infty}\left(\mathbf{R}^{v}\right)$ with $\Sigma_{0}:=\left\{x \in \mathbf{R}^{v} \mid W(x) \neq 0\right\}$ bounded.

Given (H.2.1) and (H.2.2) we define in $L^{2}\left(\mathbf{R}^{v}\right)$ :

$$
H_{0}=-\Delta+V, \quad H_{\lambda}=H_{0}+\lambda W, \quad \lambda \in \mathbf{R},
$$

where $\Delta$ denotes the usual Laplacian on the standard $L^{2}$-Sobolev space $H^{2}\left(\mathbf{R}^{v}\right)$.

Next we introduce the Dirichlet Laplacian on an arbitrary set $\mathscr{M} \subseteq \mathbf{R}^{v}$ as follows: Let $H^{1}\left(\mathbf{R}^{v}\right)$ denote the standard $L^{2}$-Sobolev space and introduce

and

$$
Q\left(-\Delta_{D}^{\mathscr{M}}\right):=\left\{g \in H^{1}\left(\mathbf{R}^{v}\right)|g|_{\mathbf{R}^{v} \backslash \mathscr{M}}=0 \text { a.e. }\right\}
$$

$$
\mathscr{H}_{D}^{\mathscr{M}}=\overline{Q\left(-\Delta_{D}^{\mathscr{M}}\right)}
$$

Now the Dirichlet Laplacian $-\Delta_{D}^{\mathscr{M}}$, as an operator in $\mathscr{H}_{D}^{\mathscr{M}}$, is defined as the operator with quadratic form domain $Q\left(-\Delta_{D}^{\mathscr{M}}\right)$ and form

$$
h(g):=\|\nabla g\|_{2}^{2}, \quad g \in Q\left(-\Delta_{D}^{\mathscr{M}}\right) .
$$

We also define in $\mathscr{H}_{D}^{\mathbf{R}^{v} \backslash \Sigma_{0}}$

$$
H_{\infty}=-\Delta_{D}^{\mathbf{R}^{v} \backslash \Sigma_{0}}+V
$$

and

$$
\tilde{R}_{\infty}(z)=\left(H_{\infty}-z\right)^{-1} \oplus 0 \quad \text { on } \quad \mathscr{H}_{D}^{\mathbf{R}^{v} \backslash \Sigma_{0}} \oplus\left\{L^{2}\left(\mathbf{R}^{v}\right) \cap\left(\mathscr{H}_{D}^{\mathbf{R}^{v} \backslash \Sigma_{0}}\right)^{\perp}\right\}, \quad z \in \mathbf{C} \backslash \sigma\left(H_{\infty}\right) .
$$

Under minimal regularity conditions on $\Sigma_{0}$, e.g. $\left|\partial \Sigma_{0}\right|=0$ and $\mathbf{R}^{v} \backslash \overline{\Sigma_{0}}$ obeying a cone condition, one can show that $C_{0}^{\infty}\left(\mathbf{R}^{v} \backslash \overline{\Sigma_{0}}\right)$ is dense in $Q\left(-\Delta_{D}^{\mathbf{R}^{v} \backslash \Sigma_{0}}\right)$ and 
one gets equality with the more conventional Dirichlet Laplacian [26].

The following is essentially in Kato [18]:

Theorem 2.1. Assume (H.2.1) and (H.2.2). Then, as $\lambda \rightarrow \infty$, the resolvent $\left(H_{\lambda}-z\right)^{-1}$ converges in norm to the pseudoresolvent $\tilde{R}_{\infty}(z)$

$$
n-\lim _{\lambda \rightarrow \infty}\left(H_{\lambda}-z\right)^{-1}=\tilde{R}_{\infty}(z), \quad z \in \mathbf{C} \backslash \sigma\left(H_{\infty}\right) .
$$

Proof. By the monotone convergence of forms [19], slightly generalized $[29,30]$, we obtain strong convergence of $\left(H_{\lambda}-z\right)^{-1}$ to $\widetilde{R}_{\infty}(z)$ since

$$
Q\left(-\Delta_{D}^{\mathbf{R}^{v} \backslash \Sigma_{0}}\right)=\left\{g \in H^{1}\left(\mathbf{R}^{v}\right) \mid(g, W g)=0\right\} .
$$

Next let $-c>0$ be large enough such that $H_{0} \geqq c+1$. Define in $L^{2}\left(\mathbf{R}^{v}\right)$

$$
C_{\lambda}:=\left(H_{\lambda}-c\right)^{-1}-\tilde{R}_{\infty}(c), \quad \lambda \geqq 0 .
$$

Then

$$
0 \leqq C_{\lambda} \leqq C_{0}, \quad \lambda \geqq 0
$$

Norm convergence now follows from two facts:

(i) $C_{0}$ is compact as follows from standard arguments $[5,18]$.

(ii) Let $C$ be a compact operator in a (separable, complex) Hilbert space and $\left\{A_{n}\right\}_{n \in N}$ a sequence of operators satisfying $0 \leqq A_{n} \leqq C, n \in \mathbf{N}$ and $s-\lim _{n \rightarrow \infty} A_{n}=A$.

Then $\lim _{n \rightarrow \infty}\left\|A_{n}-A\right\|=0$ (see, e.g. [19], p. 455).

Remark 2.2. The pseudoresolvent (cf. e.g. $[15,32]) \widetilde{R}_{\infty}(z)$ formally is the resolvent of an improper operator of the type $H_{\infty} \oplus\{\infty\}$. These improper operators are the mathematical realization of the physicists' notion of operators with hard cores (in our case the hard core is $\Sigma_{0}$ ). The standard calculus of self-adjoint operators and quadratic forms can be extended to improper ones, as discussed in $[16,17,25,29,30]$.

Remark 2.3. By using the ideas of [5] (who consider the case $W=\chi_{\Sigma_{0}}, \chi_{\mathscr{N}}$ being the characteristic function of the subset $\mathscr{N} \subset \mathbf{R}^{v}$ ), one can show that $e^{-t H_{\lambda}} \underset{\lambda \rightarrow \infty}{\longrightarrow} e^{-t H_{\infty}} \oplus 0$ in trace norm.

Theorem 2.1 then yields our main result for this section. Let $\sigma_{\text {ess }}(\cdot)$ and $\sigma_{d}(\cdot)$ denote the essential and discrete spectra, respectively.

Theorem 2.4. Assume (H.2.1) and (H.2.2), and choose an interval $[a, b] \subset \mathbf{R} \backslash \sigma_{\mathrm{ess}}\left(H_{0}\right)$. Let $\mathscr{E}_{\infty}:=\sigma\left(H_{\infty}\right) \cap(a, b)$ denote the set of all (discrete) eigenvalues of $H_{\infty}$ in $(a, b)$. Then

(i) $\sigma\left(H_{\infty}\right)=\lim _{\lambda \rightarrow \infty} \sigma\left(H_{\lambda}\right)$.

(ii) $\sigma_{\mathrm{ess}}\left(H_{\infty}\right)=\lim _{\lambda \rightarrow \infty} \sigma_{\mathrm{ess}}\left(H_{\lambda}\right)=\sigma_{\mathrm{ess}}\left(H_{0}\right)$.

(iii) For any $E_{\infty} \in \mathscr{E}_{\infty}$, there exist $E_{\lambda} \in\left(a, E_{\infty}\right) \cap \sigma_{d}\left(H_{\lambda}\right)$ such that $E_{\lambda} \uparrow E_{\infty}$ as $\lambda \uparrow \infty$. If $m_{\infty}$ denotes the multiplicity of $E_{\infty}$ then, for $\lambda>0$ large enough, there are precisely $m_{\infty}$ eigenvalues $E_{\lambda}$ of $H_{\lambda}$ (counting multiplicities) near $E_{\infty}$. 
Proof. (i) and (ii) simply follow from the strong spectral mapping theorem [26] together with Theorem 9.5 of [35]. The second equality of (2.12) is a consequence of the fact that $W$ is relatively compact with respect to $H_{0}$. The assertions in (iii) are a simple consequence of (2.7): First of all we recall that all eigenvalues of $H_{\lambda}$ in $(a, b)$ are monotonically increasing with respect to $\lambda$. Moreover, by (2.7), spectral projections $P_{H_{\lambda}}((c, d))$ of $H_{\lambda}$ converge in norm to the projection $P_{H_{\infty}}((c, d)) \oplus 0$ associated with $H_{\infty} \oplus\{\infty\}$ as $\lambda \rightarrow \infty$ by choosing $c, d$ sufficiently close to $E_{\infty}$ such that $\sigma\left(H_{\infty}\right) \cap(c, d)=\left\{E_{\infty}\right\}$,

In particular, for $\lambda>0$ large enough,

$$
\left\|P_{H_{\lambda}}((c, d))-P_{H_{\infty}}((c, d)) \oplus 0\right\| \underset{\lambda \rightarrow \infty}{\longrightarrow} 0 .
$$

$$
\operatorname{rank}\left[P_{H_{\lambda}}((c, d))\right]=\operatorname{rank}\left[P_{H_{\infty}}((c, d))\right]=m_{\infty},
$$

concluding the proof.

Remark 2.5. By a standard procedure one could relax the conditions $V, W \in L^{\infty}\left(\mathbf{R}^{v}\right)$ and admit local singularities [26].

Remark 2.6. As $\lambda \rightarrow \infty$, the eigenvalues of $H_{\lambda}$ either converge to elements of $\mathscr{E}_{\infty}$ or, if $\mathscr{E}_{\infty}=\emptyset, \sigma\left(H_{\lambda}\right) \cap(a, b)=\emptyset$ for $\lambda>0$ large enough. In either case the BirmanSchwinger kernel $W^{1 / 2}\left(H_{0}-E\right)^{-1} W^{1 / 2}, E \in(a, b)$ has only a finite number of negative eigenvalues. (By compactness it has infinitely many positive eigenvalues accumulating at zero.) This is only apparently in conflict with the completeness result in $[2,8,13,20]$ (see also [10]), where for all $E \in(a, b)$ an infinite sequence $\lambda_{j} \rightarrow \infty$ (depending on $E$ ) with the property $E \in \sigma_{d}\left(H_{\lambda_{j}}\right), j \in \mathbf{N}$ has been established. The reason is that our condition $W \geqq 0$ explicitly violates the crucial assumption $W_{-}(x) \geqq \mu>0$ for $x$ in some open ball of radius $\varepsilon>0$ in $[2,8,10,13]$. (Here $W_{ \pm}(x):=[|W(x)| \pm W(x)] / 2$ denote the positive and negative parts of $W$.)

\section{The Repulsive Case in One Dimensions}

In this section we illustrate the results of the foregoing section by means of one-dimensional considerations. We also estimate the rate at which the eigenvalues of $H_{\lambda}$ converge to those of $H_{\infty}$.

Example 3.1. Let $V \in L^{\infty}(\mathbf{R})$ be real-valued and define $H_{0}=-d^{2} / d x^{2}+V$ in $L^{2}(\mathbf{R})$. Assume $E_{\infty} \notin \sigma_{\text {ess }}\left(H_{0}\right)$ and $E_{\infty}>\inf \left(\sigma_{\text {ess }}\left(H_{0}\right)\right)$. By Weyl's limit point/circle classification there exist two (linearly independent) real solutions $f_{0, \pm}\left(E_{\infty}, x\right)$ such that

$$
H_{0} f_{0, \pm}=E_{\infty} f_{0, \pm} \text { (distributional sense), } f_{0, \pm} \in L^{2}([R, \pm \infty)), \text { for all } R \in \mathbf{R} \text {. }
$$

Since $E_{\infty}>\inf \left(\sigma_{\text {ess }}\left(H_{0}\right)\right)$, oscillation theorems [9] imply that both $f_{0, \pm}$ have infinitely many zeroes on $\mathbf{R}$. Choose $x_{+}$such that $f_{0,+}\left(E_{\infty}, x_{+}\right)=0$, let $x_{-}<x_{+}$, and define $W(x)=\chi_{\left[x_{-}, x_{+}\right]}(x)$ and $H_{\lambda}=H_{0}+\lambda W, \lambda>0$ in $L^{2}(\mathbf{R})$. Then obviously Theorem 2.1 applies. In particular, we have the direct sum decomposition

$$
\begin{array}{ll}
L^{2}(\mathbf{R} \backslash \Sigma)=L^{2}\left(\left(-\infty, x_{-}\right)\right) \oplus L^{2}\left(\left(x_{+}, \infty\right)\right), & \\
H_{\infty}=-\Delta_{D}^{\mathbf{R} \backslash \Sigma}+V=H_{-} \oplus H_{+}, & \Sigma=\left[x_{-}, x_{+}\right],
\end{array}
$$


where

$$
\begin{gathered}
H_{ \pm}=-\Delta_{D}^{\left(x_{ \pm}, \pm \infty\right)}+V \\
-\Delta_{D}^{\left(x_{ \pm}, \pm \infty\right)}=-\frac{d^{2}}{d x^{2}}, \quad \mathscr{D}\left(-\Delta_{D}^{\left(x_{ \pm}, \pm \infty\right)}\right)=\left\{g \in H^{2}\left(\left(x_{ \pm}, \pm \infty\right)\right) \mid g\left(x_{ \pm}\right)=0\right\} .
\end{gathered}
$$

Define $f_{ \pm}:=\left.f_{0, \pm}\right|_{\left(x_{+}, \pm \infty\right)}$. By construction, $H_{+} f_{+}=E_{\infty} f_{+}, f_{+} \in \mathscr{D}\left(H_{+}\right)$. If, by accident, we also have $f_{-}\left(E_{\infty}, x_{-}\right)=0$, then $H_{-} f_{-}=E_{\infty} f_{-}, f_{-} \in \mathscr{D}\left(H_{-}\right)$, and so $E_{\infty} \in \sigma_{d}\left(H_{\infty}\right)$ has multiplicity two. Otherwise (i.e. if $\left.f_{-}\left(E_{\infty}, x_{-}\right) \neq 0\right), E_{\infty} \in \sigma_{d}\left(H_{\infty}\right)$ is simple. In either case Theorem 2.6 applies.

\section{Remark 3.2.}

a) Clearly the above construction fails if $E_{\infty}<\inf \left(\sigma\left(H_{0}\right)\right)$ since then both $f_{0, \pm}\left(E_{\infty}, x\right), x \in \mathbf{R}$ have no zeroes at all.

b) If $V$ is periodic, (i.e., for some $a>0, V(x+a)=V(x)$, for all $x$ ), then Floquet theory implies

$$
f_{0, \pm}\left(E_{\infty, x}\right)=e^{\mp \alpha x} P_{ \pm}(x), \quad \alpha>0, \quad P_{ \pm}(x+a)=P_{ \pm}(x)
$$

and generically $H_{0}$ has infinitely many spectral gaps.

c) Although $H_{\infty}$ might have degenerate eigenvalues as explained above, $H_{\lambda}$ has only simple eigenvalues since Theorem II.1.5 of [28] applies.

d) Instead of the projection $\chi_{\Sigma}$ in $H_{\lambda}$, we may take any $0 \leqq W \in L^{\infty}(\mathbf{R})$ of suitable compact support. For simplicity assume that $\operatorname{supp}(W)$ consists of a finite union of compact intervals with $x_{-}:=\inf (\operatorname{supp}(W)), x_{+}:=\sup (\operatorname{supp}(W))$. Then simply translate $W$ as a whole to the left or right (keeping the background potential $V$ fixed) such that $x_{-}$or $x_{+}$coincide with one of the (infinitely many) zeroes of $f_{0,+}\left(\right.$ or $\left.f_{0,-}\right)$. Then again $E_{\infty} \in \sigma_{d}\left(H_{\infty}\right)$ and the above construction works.

e) At first sight one might think that the above example extends to $v \geqq 2$ dimensions by choosing a spherically symmetric potential. However, the results of [14] show that the spectral gaps of the radially symmetric, one-dimensional operators on the half-line $(0, \infty)$ associated with a fixed angular momentum, disappear in the full Schrödinger operator in $L^{2}\left(\mathbf{R}^{v}\right)$ (the direct sum over all angular momenta).

f) The condition $V \in L^{\infty}(\mathbf{R})$ in Example 3.1 is too restrictive. It can be replaced by $V \in L_{\text {loc }}^{1}(\mathbf{R})$ and the additional assumption that the differential expression $-d^{2} / d x^{2}+V$ is in the limit point case at $\pm \infty$.

We add a few remarks on Dirichlet operators in one dimension. Consider in $L^{2}(\mathbf{R})=L^{2}(\mathbf{R} \backslash \Sigma) \oplus L^{2}(\Sigma), \Sigma=\left[x_{-}, x_{+}\right], x_{-}<x_{+}$the Dirichlet Hamiltonian

$$
\begin{aligned}
& H_{\infty, D}=-\Delta_{D}+V=H_{\infty} \oplus H_{-,+}=H_{-} \oplus H_{+} \oplus H_{-,+}, \\
& -\Delta_{D}=-\frac{d^{2}}{d x^{2}}, \quad \mathscr{D}\left(-\Delta_{D}\right)=\left\{g \in H^{1}(\mathbf{R}) \cap H^{2}\left(\mathbf{R} \backslash\left\{x_{-}, x_{+}\right\}\right) \mid g\left(x_{ \pm}\right)=0\right\}, \\
& H_{-,+}=-\Delta_{D}^{\Sigma^{0}}+V \quad \text { in } \quad L^{2}(\Sigma), \quad \Sigma^{0}=\left(x_{-}, x_{+}\right) .
\end{aligned}
$$

Define

$$
R_{0}(z)=\left(H_{0}-z\right)^{-1}, \quad z \in \mathbf{C} \backslash \sigma\left(H_{0}\right),
$$




$$
\begin{aligned}
R_{0}\left(z, x, x^{\prime}\right) & =\left[W\left(f_{0,-}(z), f_{0,+}(z)\right)\right]^{-1} \begin{cases}f_{0,-}(z, x) f_{0,+}\left(z, x^{\prime}\right), & x \leqq x^{\prime} \\
f_{0,-}\left(z, x^{\prime}\right) f_{0,+}(z, x), & x \geqq x^{\prime}\end{cases} \\
R_{\infty, D}(z) & =\left(H_{\infty, D}-z\right)^{-1}, \quad z \in \mathbf{C} \backslash \sigma\left(H_{\infty, D}\right),
\end{aligned}
$$

where $W(f, g)=\bar{f} g^{\prime}-\bar{f}^{\prime} g$ denotes the Wronskian of $f$ and $g$. Then, by Krein's theory [9], the corresponding integral kernel of $R_{\infty, D}(z)$ satisfies

$$
R_{\infty, D}\left(z, x, x^{\prime}\right)=R_{0}\left(z, x, x^{\prime}\right)-\sum_{j, j^{\prime}= \pm}[\gamma(z)]_{j j^{\prime}}^{-1} R_{0}\left(z, x, x_{j^{\prime}}\right) R_{0}\left(z, x_{j}, x^{\prime}\right),
$$

where

$$
\gamma(z)=\left[\begin{array}{ll}
R_{0}\left(z, x_{-}, x_{-}\right) & R_{0}\left(z, x_{+}, x_{-}\right) \\
R_{0}\left(z, x_{-}, x_{+}\right) & R_{0}\left(z, x_{+}, x_{+}\right)
\end{array}\right] .
$$

In spectral gaps of $H_{0}, W\left(f_{0,-}(E), f_{0,+}(E)\right) \neq 0, E \in \mathbf{R} \backslash \sigma\left(H_{0}\right)$ and the eigenvalues of $H_{\infty, D}$ in $\mathbf{R} \backslash \sigma\left(H_{0}\right)$ are given by

$$
\begin{aligned}
\operatorname{det}[\gamma(E)]= & 0 \Leftrightarrow f_{0,-}\left(E, x_{-}\right) f_{0,+}\left(E, x_{+}\right)\left\{f_{0,+}\left(E, x_{-}\right) f_{0,-}\left(E, x_{-}\right)\right. \\
& \left.-f_{0,+}\left(E, x_{+}\right) f_{0,-}\left(E, x_{-}\right)\right\}=0 .
\end{aligned}
$$

Clearly $f_{0, \pm}\left(E, x_{ \pm}\right)=0$ yields the eigenvalues of $H_{ \pm}$, whereas $\{\cdots\}=0$ in (3.13) is responsible for those of $H_{-,+}$in $\mathbf{R} \backslash \sigma\left(H_{0}\right)$. Krein's theory implies that $H_{\infty, D}$ can have at most two eigenvalues (counting multiplicity) in each spectral gap of $H_{0}$. (Since $H_{\infty, D} \geqq H_{0}$, there are no eigenvalues of $H_{\infty, D}$ below $\inf \left(\sigma\left(H_{0}\right)\right)$.)

In the special case where $x_{-}, x_{+} \rightarrow x_{0}$, i.e. where the support of $W$ shrinks to the point $x_{0}$ (cf. Sect. 7), one considers

$$
\begin{aligned}
H_{\infty} & =-\Delta_{D}^{\mathbf{R} \backslash\left\{x_{0}\right\}}+V, \\
-\Delta_{D}^{\mathbf{R} \backslash\left\{x_{0}\right\}} & =-\frac{d^{2}}{d x^{2}}, \quad \mathscr{D}\left(-\Delta_{D}^{\mathbf{R} \backslash\left\{x_{0}\right\}}\right)=\left\{g \in H^{1}(\mathbf{R}) \cap H^{2}\left(\mathbf{R} \backslash\left\{x_{0}\right\}\right) \mid g\left(x_{0}\right)=0\right\} .
\end{aligned}
$$

Let

then

$$
R_{\infty}(z)=\left(H_{\infty}-z\right)^{-1}, \quad z \in \mathbf{C} \backslash \sigma\left(H_{\infty}\right) .
$$

$$
R_{\infty}\left(z, x, x^{\prime}\right)=R_{0}\left(z, x, x^{\prime}\right)-\frac{R_{0}\left(z, x, x_{0}\right) R_{0}\left(z, x_{0}, x^{\prime}\right)}{R_{0}\left(z, x_{0}, x_{0}\right)} .
$$

Thus the eigenvalues of $H_{\infty}$ in $\mathbf{R} \backslash \sigma\left(H_{0}\right)$ are given by

$$
R_{0}\left(E, x_{0}, x_{0}\right)=0 \Leftrightarrow f_{0,-}\left(E, x_{0}\right) f_{0,+}\left(E, x_{0}\right)=0 .
$$

Since $W\left(f_{0,-}(E), f_{0,+}(E)\right) \neq 0$ for $E \in \mathbf{R} \backslash \sigma\left(H_{0}\right),(3.17)$ is equivalent to

$$
\text { either } f_{0,-}\left(E, x_{0}\right)=0 \text { or } f_{0,+}\left(E, x_{0}\right)=0 \text { but not both. }
$$

(In particular, if $V$ is reflection symmetric about $x_{0}$ then $f_{0 .-}\left(E, x_{0}\right)=0=$ $f_{0,+}\left(E, x_{0}\right), E \in \mathbf{R} \backslash \sigma\left(H_{0}\right)$ would violate (3.18). Thus $\sigma_{p}\left(H_{\infty}\right) \cap\left[\mathbf{R} \backslash \sigma\left(H_{0}\right)\right]=\emptyset$ in this special case.) In general, Krein's theory shows that $H_{\infty}$ has at most one, simple eigenvalue in each spectral gap of $H_{0}$ (except in $\left(-\infty, \inf \left[\sigma\left(H_{0}\right)\right]\right)$, since $\left.H_{\infty} \geqq H_{0}\right)$. 
These remarks can easily be generalized to the case where spectral gaps of $H_{0}$ are replaced by essential spectral gaps of $H_{0}$ using the Weinstein-Aronszajn formulas [19].

Finally we describe the rate at which eigenvalues of $H_{\lambda}$ converge to those of $H_{\infty}$, employing quassi-classical methods. We introduce

(H.3.1) Assume $V \in C^{0}(\mathbf{R})$ with the differential expression $-d^{2} / d x^{2}+V$ in the limit-point case at $\pm \infty$.

(H.3.2) $0<\varepsilon \leqq W \in C^{2}((0, l)), \quad \Sigma:=\operatorname{supp}(W)=[0, l], \quad l>0 \quad$ (i.e. square-well-like potentials $W$ ), or

(H.3.3) $0<\varepsilon \leqq \tilde{W} \in C^{2}((0, l)), l>0, W(x)=x(l-x) \tilde{W}(x), x \in(0, l), \Sigma:=\operatorname{supp}(W)=$ $[0, l]$ (i.e. potentials $W$ with simple turning points at $x=0, l$ ).

Given (H.3.1) and (H.3.2) or (H.3.1) and (H.3.3) we define $H_{0}, H_{\lambda}, \lambda>0$ in $L^{2}(\mathbf{R})$ and $H_{\infty}$ in $L^{2}(\mathbf{R} \backslash \Sigma)$ as in Example 3.1. Finally, we introduce

(H.3.4) Assume $[a, b] \subset \mathbf{R} \backslash \sigma_{\text {ess }}\left(H_{0}\right)$ and let $\mathscr{E}_{\infty}:=\sigma\left(H_{\infty}\right) \cap(a, b)$ be the (possibly empty) set of (discrete) eigenvalues of the Dirichlet operator $H_{\infty}$ in $(a, b)$.

Theorem 3.3. Assume (H.3.1), (H.3.2) or (H.3.3), and (H.3.4).

(i) Suppose $E_{\infty} \in \mathscr{E}_{\infty}$ with multiplicity $m_{\infty}\left(m_{\infty}=1,2\right)$. Then, as $\lambda \uparrow \infty, H_{\lambda}$ has precisely $m_{\infty}$ distinct and simple eigenvalues $E_{j, \lambda}$ below $E_{\infty}$ such that,

$$
E_{j, \lambda}=E_{\infty}-c_{j} \lambda^{-\alpha}+O\left(\lambda^{-2 \alpha}\right), \quad 1 \leqq j \leqq m_{\infty} .
$$

Here $\alpha=1 / 2$ in the square-well-like case (H.3.2), while $\alpha=1 / 3$ in the turningpoint case (H.3.3). (The positive coefficients $c_{j}$ are described in the proof.) Except for these eigenvalues $E_{j, \lambda}$ near $E_{\infty}, H_{\lambda}$ has no other eigenvalues in $(a, b)$ for $\lambda>0$ large enough (cf. Fig. 3.1).

(ii) If $\mathscr{E}_{\infty}=\emptyset$, then $\sigma\left(H_{\lambda}\right) \cap(a, b)=\emptyset$ for $\lambda>0$ sufficiently large.

Proof. The convergence $E_{\lambda} \uparrow E_{\infty}$ follows from Theorem 2.5 and Remark 3.2c. What remains to be proven is (3.19).

a) Assume (H.3.2) (i.e. the square-well-like case). An eigenfunction $\psi(E, x, \lambda)$ of $H_{\lambda}$ must take the form:

$$
\psi(E, x, \lambda)= \begin{cases}C_{-}(E, \lambda) f_{-}(E, x), & x<0 \\ A(E, \lambda) \Phi_{+}(E, x, \mu)+B(E, \lambda) \Phi_{-}(E, x, \mu), & 0<x<l, \\ C_{+}(E, \lambda) f_{+}(E, x), & x>l\end{cases}
$$

where $\Phi_{ \pm}$are linearly independent solutions of the Schrödinger equation on $[0, l]$. By WKB theory [22] we can take

$$
\begin{aligned}
& \Phi_{-}=W(x)^{-1 / 4} \exp \left[-\mu \int_{0}^{x} \sqrt{W(t)} d t\right]\left[1+O\left(\mu^{-1}\right)\right] \\
& \Phi_{+}=W(x)^{-1 / 4} \exp \left[-\mu \int_{x}^{l} \sqrt{W(t)} d t\right]\left[1+O\left(\mu^{-1}\right)\right]
\end{aligned}
$$

where $\mu=\sqrt{\lambda}$, with $A, B, C_{ \pm}$appropriate constants and $f_{ \pm}(E, x)$ as in Example 3.1 . 


\section{Define}

$$
\begin{aligned}
h_{-}(E) & :=\frac{f_{-}\left(E, 0_{-}\right)}{f_{-}^{\prime}\left(E, 0_{-}\right)}, \quad h_{+}(E):=\frac{f_{+}\left(E, l_{+}\right)}{f_{+}^{\prime}\left(E, l_{+}\right)}, \\
W_{0} & :=\lim _{x \rightarrow 0^{+}} W(x), \quad W_{0}^{\prime}:=\lim _{x \rightarrow 0^{+}} W^{\prime}(x), \\
W_{l}: & =\lim _{x \rightarrow l_{-}} W(x), \quad W_{l}^{\prime}:=\lim _{x \rightarrow l_{-}} W^{\prime}(x) .
\end{aligned}
$$

Setting $\psi=h_{-} \psi^{\prime}$ at $x=0$ and $\psi=h_{+} \psi^{\prime}$ at $x=l$ gives the system of linear equations

$$
\left(\begin{array}{ll}
\Phi_{+}(0)-h_{-} \Phi_{+}^{\prime}(0) & \Phi_{-}(0)-h_{-} \Phi_{-}^{\prime}(0) \\
\Phi_{+}(l)-h_{+} \Phi_{+}^{\prime}(l) & \Phi_{-}(l)-h_{+} \Phi_{-}^{\prime}(l)
\end{array}\right)\left(\begin{array}{l}
A \\
B
\end{array}\right)=0 .
$$

Setting the determinant equal to zero gives

$$
\begin{aligned}
& \frac{\left\{h_{-}(E)\left[\sqrt{W_{0}}+O\left(\mu^{-1}\right)\right]+\mu^{-1}\right\}\left\{h_{+}(E)\left[\sqrt{W_{l}}+O\left(\mu^{-1}\right)\right]-\mu^{-1}\right\}}{\left\{h_{-}(E)\left[\sqrt{W_{0}}+O\left(\mu^{-1}\right)\right]-\mu^{-1}\right\}\left\{h_{+}(E)\left[\sqrt{W_{l}}+O\left(\mu^{-1}\right)\right]+\mu^{-1}\right\}} \\
& \quad=\left[1+O\left(\mu^{-1}\right)\right] e^{-2 \mu L}
\end{aligned}
$$

as $\mu \rightarrow \infty$, where $L:=\int_{0}^{l} \sqrt{W(t)} d t$. Consequently, either

or

$$
h_{-}(E)=-\mu^{-1} W_{0}^{-1 / 2}+O\left(\mu^{-2}\right)
$$

$$
h_{+}(E)=\mu^{-1} W_{l}^{-1 / 2}+O\left(\mu^{-2}\right) .
$$

(This can be alternately understood from the fact that either $A \gg B e^{-\mu L}$, in which case (3.25) holds, or $B \gg A e^{-\mu L}$, in which case (3.26) holds.)

In the first case, $E$ must be close to an eigenvalue $E_{\infty,-}$ of $H_{-}$(cf. (3.3)), and hence by analyticity of $f_{ \pm}(E, x)$ with respect to $E$,

$$
h_{-}(E)=d_{-}\left(E-E_{\infty,-}\right)+O\left(\left(E-E_{\infty,-}\right)^{2}\right) \text {, }
$$

whereas in the second case $E$ must be close to an eigenvalue $E_{\infty,+}$ of $H_{+}$(cf. (3.3)) and hence

$$
h_{+}(E)=d_{+}\left(E-E_{\infty,+}\right)+O\left(\left(E-E_{\infty,+}\right)^{2}\right) .
$$

By general principles, $d_{+}<0<d_{-}$, as taking the Wronskian $W_{+}(x):=W\left(f_{+}(E)\right.$, $\left.f_{+}\left(E_{\infty,+}\right)\right)(x)$ and integrating the identity $W_{+}^{\prime}(x)=\left(E-E_{\infty,+}\right) f_{+}(E, x) f_{+}\left(E_{\infty,+}, x\right)$ from $l$ to $\infty$ yields

$$
h_{+}(E)=-\left\{\int_{l}^{\infty} d x\left(\frac{f_{+}\left(E_{\infty,+}, x\right)}{f_{+}^{\prime}\left(E_{\infty,+}, l_{+}\right)}\right)^{2}\right\}\left(E-E_{\infty,+}\right)+O\left(\left(E-E_{\infty,+}\right)^{2}\right),
$$

i.e., $d_{+}<0$. Similarly, one shows that $d_{-}>0$. Thus we infer that, as $\mu \rightarrow \infty$, either

$$
E(\mu)=E_{\infty,--}-\left[\mu d_{-} W_{0}^{1 / 2}\right]^{-1}+O\left(\mu^{-2}\right)
$$

or

$$
E(\mu)=E_{\infty,+}+\left[\mu d_{+} E_{l}^{1 / 2}\right]^{-1}+O\left(\mu^{-2}\right),
$$

where $E(\mu) \equiv E_{\lambda}$. In the case of a degenerate Dirichlet eigenvalue $E_{\infty,-}=E_{\infty,+}$, 
both possibilities in (3.30) and (3.31) are realized. This proves (3.19) with $\alpha=1 / 2$. b) Assume (H.3.3) (i.e. the turning-point case). The procedure here is similar to the previous case, but the analysis is more involved, as the WKB approximation (3.21) is no longer valid near the turning points $x=0$ and $x=l$. Near 0 and $l$ we must approximate $\Phi_{ \pm}$by Airy functions (solutions of the Airy equation $y^{\prime \prime}=x y$ ), and match these to the interior solutions (3.21). This procedure is standard [22], and we merely quote the results:

$$
\begin{aligned}
& \Phi_{-}(x)=\left\{\begin{array}{l}
2 \sqrt{\pi} \lambda^{1 / 3} W_{0}^{\prime-1 / 6} \operatorname{Ai}\left(\left[\lambda W_{0}^{\prime}\right]^{1 / 3} x\right)\left(1+O\left(\lambda^{-1 / 3}\right)\right) \quad \text { near } x=0 \\
W(x)^{-1 / 4} \exp \left(-\mu \int_{0}^{x} \sqrt{W}\right)\left(1+O\left(\lambda^{-1 / 2}\right)\right) \quad \text { away from } 0 \text { and } l, \\
e^{-\mu L} \sqrt{\pi} \lambda^{1 / 3}\left|W_{l}^{\prime}\right|^{-1 / 6} \operatorname{Bi}\left(\left|\lambda W_{l}^{\prime}\right|^{1 / 3}(l-x)\right)\left(1+O\left(\lambda^{-1 / 3}\right)\right) \quad \text { near } x=l
\end{array}\right. \\
& \Phi_{+}(x)=\left\{\begin{array}{l}
e^{-\mu L} \sqrt{\pi} \lambda^{1 / 3} W_{0}^{\prime-1 / 6} \operatorname{Bi}\left(\left[\lambda W_{0}^{\prime}\right]^{1 / 3} x\right)\left(1+O\left(\lambda^{-1 / 3}\right)\right) \quad \text { near } x=0 \\
W(x)^{-1 / 4} \exp \left(-\mu \int_{x}^{l} \sqrt{W}\right)\left(1+O\left(\lambda^{-1 / 2}\right)\right) \quad \text { away from } 0 \text { and } l, \\
2 \sqrt{\pi} \lambda^{1 / 3}\left|W_{l}^{\prime}\right|^{-1 / 6} \operatorname{Ai}\left(\left|\lambda W_{l}^{\prime}\right|^{1 / 3}(l-x)\right)\left(1+O\left(\lambda^{-1 / 3}\right)\right) \quad \text { near } x=l
\end{array}\right.
\end{aligned}
$$

where $\mathrm{Ai}(z)$ and $\mathrm{Bi}(z)$ are Airy functions of the first and second kind, respectively $[1,22]$. The Wronskian matrix of the Airy functions at 0 is

$$
\left(\begin{array}{cc}
\mathrm{Ai}(0) & \mathrm{Bi}(0) \\
\mathrm{Ai}^{\prime}(0) & \mathrm{Bi}^{\prime}(0)
\end{array}\right)=\left(\begin{array}{cl}
1 /\left\{3^{2 / 3} \Gamma(2 / 3)\right\} & 1 /\left\{3^{1 / 6} \Gamma(2 / 3)\right\} \\
-1 /\left\{3^{1 / 3} \Gamma(1 / 3)\right\} & 3^{1 / 6} /\{\Gamma(1 / 3)\}
\end{array}\right) .
$$

As with the square-well-like case, we get a system of linear equations (3.23), and we may set the determinant of the matrix equal to zero, getting

$$
\begin{aligned}
& \frac{\left\{h_{-}(E)\left[\left(W_{0}^{\prime}\right)^{1 / 3}+O\left(\lambda^{-1 / 3}\right)\right]+\omega \lambda^{-1 / 3}\right\}\left\{h_{+}(E)\left[\left|W_{l}^{\prime}\right|^{1 / 3}+O\left(\lambda^{-1 / 3}\right)\right]-\omega \lambda^{-1 / 3}\right\}}{\left\{h_{-}(E)\left[\left(W_{0}^{\prime}\right)^{1 / 3}+O\left(\lambda^{-1 / 3}\right)\right]-\omega \lambda^{-1 / 3}\right\}\left\{h_{+}(E)\left[\left|W_{l}^{\prime}\right|^{1 / 3}+O\left(\lambda^{-1 / 3}\right)\right]+\omega \lambda^{-1 / 3}\right\}} \\
& \quad=\frac{3 e^{-2 \mu L}}{4}\left[1+O\left(\mu^{-1}\right)\right],
\end{aligned}
$$

where $\omega=3^{-1 / 3} \Gamma\left(\frac{1}{3}\right) / \Gamma\left(\frac{2}{3}\right)$. This implies that either

$$
h_{-}=-\omega\left|W_{0}^{\prime} \lambda\right|^{-1 / 3}+O\left(\lambda^{-2 / 3}\right) \text {, }
$$

or

$$
h_{+}=\omega\left|W_{l}^{\prime} \lambda\right|^{-1 / 3}+O\left(\lambda^{-2 / 3}\right) \text {. }
$$

(If $A \gg B e^{-\mu L}$, then $h_{-} \approx \Phi_{+}(0) / \Phi_{+}^{\prime}(0)$, and (3.36) applies. Otherwise, $B \gg A e^{-\mu L}$, so $h_{+} \approx \Phi_{+}(l) / \Phi_{+}^{\prime}(l)$ and (3.37) applies.)

In the first case $E$ must be close to an eigenvalue $E_{\infty,-}$ of $H_{-},(3.27)$ applies, and as $\lambda \rightarrow \infty$ we have

$$
E_{\lambda}=E_{\infty,-}-\frac{\omega}{d_{-}}\left[\lambda W_{0}^{\prime}\right]^{-1 / 3}+O\left(\lambda^{-2 / 3}\right)
$$

whereas in the second case $E$ must be close to an eigenvalue $E_{\infty_{+}+}$of $H_{+},(3.28)$ 
applies, and as $\lambda \rightarrow \infty$ we have

$$
E_{\lambda}=E_{\infty,+}+\frac{\omega}{d_{+}}\left|\lambda W_{l}^{\prime}\right|^{-1 / 3}+O\left(\lambda^{-2 / 3}\right)
$$

In the case of a degenerate Dirichlet eigenvalue $E_{\infty,+}=E_{\infty,-}$, both possibilities in (3.38) and (3.39) are realized. This proves (3.19) with $\alpha=1 / 3$, and completes the proof of Theorem 3.3.

Remark 3.4. Without going into detail, we point out that $E_{j, \lambda}$ in (3.19) has an asymptotic series with respect to $\lambda^{-1 / 2}$ (respectively $\lambda^{-1 / 6}$ ) in the square-well-like case (H.3.2) (respectively turning-point case (H.3.3)).

Remark 3.5. Analyticity of $E_{\lambda}$ with respect to certain negative fractional powers of $\lambda$ has been derived in [4] for another large coupling problem, for a special class of potentials $W$ with support exterior to some bounded region in $\mathbf{R}^{v}$.

\section{Some Negative Results for Attractive Potentials}

The main purpose of this section is to show that there is no norm resolvent convergence of $H_{\lambda}$ to $H_{\infty}$ or any other limiting operator for the case $W \geqq 0$, $\lambda \rightarrow-\infty$.

Theorem 4.1. Assume (H.2.1) and (H.2.2). In addition suppose that $W \geqq \mu>0$ on some open ball $B_{\varepsilon} \subset \mathbf{R}^{v}$ of radius $\varepsilon>0$. Then $\left(H_{\lambda}-z\right)^{-1}$ does not converge in norm as $\lambda \rightarrow-\infty$.

Proof. Fix an arbitrary $E_{0}<-\|V\|_{\infty}$. By commutation [7],

$$
\sigma\left(W^{1 / 2}\left(H_{0}-E_{0}\right)^{-1} W^{1 / 2}\right)=\sigma\left(\left(H_{0}-E_{0}\right)^{-1 / 2} W\left(H_{0}-E_{0}\right)^{-1 / 2}\right) .
$$

Moreover,

$$
\left(H_{0}-E_{0}\right)^{-1 / 2} W\left(H_{0}-E_{0}\right)^{-1 / 2} \geqq\left(H_{0}-E_{0}\right)^{-1 / 2} \mu \chi_{B_{\varepsilon}}\left(H_{0}-E_{0}\right)^{-1 / 2} .
$$

Using commutation again and the fact that $\chi_{B_{\varepsilon}}\left(H_{0}-E_{0}\right)^{-1} \chi_{B_{\varepsilon}} \geqq \chi_{B_{\varepsilon}}(-\Delta+$ $\left.\|V\|_{\infty}-E_{0}\right)^{-1} \chi_{B_{\varepsilon}}$, and that the latter is not of finite rank, the minimax principle [26] guarantees the existence of a sequence $\lambda_{n}<0, \lambda_{n} \rightarrow-\infty$ as $n \rightarrow \infty$ such that

$$
W^{1 / 2}\left(H_{0}-E_{0}\right)^{-1} W^{1 / 2} f_{n}=-\lambda_{n}^{-1} f_{n}, \quad f_{n} \in L^{2}\left(\mathbf{R}^{v}\right), \quad n \in \mathbf{N},
$$

or equivalently,

$$
H_{\lambda_{n}} g_{n}=E_{0} g_{n}, \quad g_{n} \in L^{2}\left(\mathbf{R}^{v}\right), \quad n \in \mathbf{N} .
$$

Next assume that $\left(H_{\lambda_{n}}-z\right)^{-1}, z \in \mathbf{C} \backslash \mathbf{R}$ converges in norm as $n \rightarrow \infty$ and denote the limit by $\tilde{R}_{\infty}(z)$. Obviously $\tilde{R}_{\infty}(z)$ is a pseudoresolvent and hence there exists an orthogonal projection $P_{\infty}$ such that $\tilde{R}_{\infty}(z)=\left(H_{\infty}-z\right)^{-1} P_{\infty}$ for some self-adjoint operator $H_{\infty}$ in $P_{\infty} L^{2}\left(\mathbf{R}^{v}\right)$. By Theorem VIII.23 of [25], $E_{0} \in \sigma_{d}\left(H_{\infty}\right)$. Since $E_{0}<-\|V\|_{\infty}$ was arbitrary, we get the contradiction

$$
\left(-\infty,-\|V\|_{\infty}\right] \subset \sigma_{d}\left(H_{\infty}\right) .
$$

Remark 4.2. In the proof of Theorem 4.1 we used Theorem VIII.23 of [25], although our limit is an improper operator of the type $H_{\infty} \oplus\{\infty\}$. However, the 
corresponding proof in [25] with the limit resolvent replaced by a pseudoresolvent goes through in the same manner.

Remark 4.3. The proof of Theorem 4.1 does not immediately extend to the case where $W$ changes sign since it is not a priori clear whether $|W|^{1 / 2} \operatorname{sgn}(W)\left(H_{0}-\right.$ $\left.E_{0}\right)|W|^{1 / 2}$ (which is isospectral to $\left(H_{0}-E_{0}\right)^{-1 / 2} W\left(H_{0}-E_{0}\right)^{-1 / 2}$ ) has infinitely many negative eigenvalues. However, using the technique of [10], one can indeed derive a corresponding negative result (assuming $W_{-} \geqq \mu>0$ on some open ball of radius $\varepsilon$ ).

The results of Sects. 5 and 6 provide many examples where $H_{\lambda}$ does not even converge in strong resolvent sense to $H_{\infty}$ as $\lambda \rightarrow-\infty$.

\section{The Cascade Phenomenon in One Dimension, $W<0$}

In this section we start to look at the "cascade" phenomenon which occurs in one-dimensional systems whenever $W \geqq 0, \lambda>0$. The simpler case where $W \leqq 0$ is treated in this section, and the case where $W$ changes sign is treated in Sect. 6 . We will assume (H.3.4) throughout.

The cascade phenomenon is exhibited in Fig. 5.1, and may be described as follows: For a typical value of $\lambda>0$, the spectrum of $H_{\lambda}$ is approximately that of $H_{\infty}$. That is, there is one eigenvalue of $H_{\lambda}$ near each non-degenerate eigenvalue of $H_{\infty}$, two eigenvalues of $H_{\lambda}$ near each (twice) degenerate eigenvalue of $H_{\infty}$, and there are no other eigenvalues of $H_{\lambda}$. As $\lambda$ varies over a long interval, the eigenvalues of $H_{\lambda}$ change very slowly, and remain near the eigenvalues of $H_{\infty}$. We call these intervals of slow change "plateaus."

However, near certain values of $\lambda$, the spectrum of $H_{\lambda}$ changes drastically, as each eigenvalue of $H_{\lambda}$ shifts down by one. We call these intervals of rapid change "transition regions." Usually, each eigenvalue of $H_{\lambda}$ shifts from one Dirichlet eigenvalue to the next lower one. However, near a degenerate Dirichlet eigenvalue, there are two eigenvalues of $H_{\lambda}$. The lower one shifts down to the next Dirichlet eigenvalue, and the higher one takes the place of the lower one. In Fig. 5.1, $E_{1}, E_{2}$,

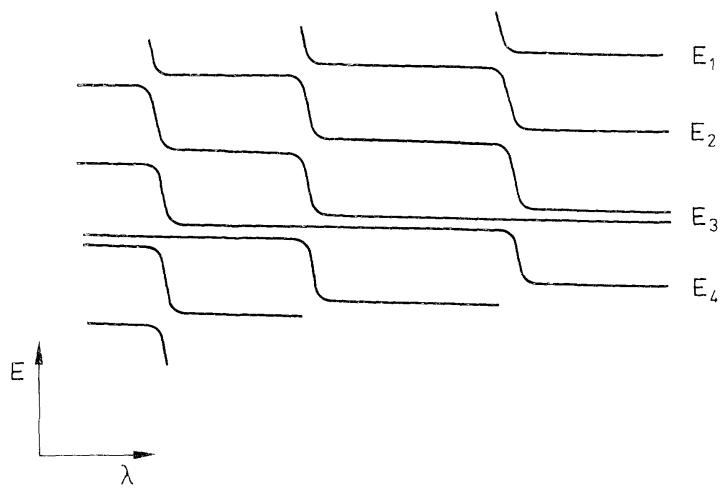

Fig. 5.1. The cascade phenomenon with a degenerate level 
and $E_{4}$ are nondegenerate Dirichlet eigenvalues, and $E_{3}$ is a degenerate Dirichlet eigenvalue.

The lengths of the plateaus grow like $\sqrt{\lambda}$ as $\lambda \rightarrow \infty$. The lengths of the transition regions, by contrast, at most grow as $\lambda^{\delta}$, where $\delta<\frac{1}{2}$ depends on the specific system. (For square-well-like potentials $W, \delta=0$; for continuous potentials with simple zeroes ("turning-point" potentials), $\delta=1 / 6$; for potentials with $n$-th order zeroes, $\delta=\frac{1}{2}-1 / n+2$.) Thus, as $\lambda \rightarrow \infty$, the system spends more and more time near the Dirichlet eigenvalues.

We make the notion of a cascade precise with the following definition:

Definition 5.1. A system is said to exhibit the cascade phenomenon on the (energy) intervals $(a, b)$ if, given a small number $\varepsilon>0$, we can choose closed intervals $T_{1}, T_{2}, \ldots$ and constants $0<\delta<\frac{1}{2}$ and $\Lambda>0$ such that, for all $\lambda>\Lambda$,

1. The size of the transition regions $T_{n}$ is bounded by a constant times $\lambda^{\delta}$, and the number of transition regions below a certain value of $\lambda$ is bounded by a constant times $\lambda^{1 / 2}$.

Let the plateau $P_{n},(n \geqq 2)$ be the open interval between $T_{n-1}$ and $T_{n}$, and let $P_{1}$ be the open interval between 0 and $T_{1}$.

2. For $\lambda \in \bigcup_{n \in \mathbf{N}} P_{n}$, there is one eigenvalue of $H_{\lambda}$ within $\varepsilon$ of each nondegenerate element of $\mathscr{E}_{\infty}$, there are two eigenvalues of $H_{\lambda}$ within $\varepsilon$ of each degenerate element of $\mathscr{E}_{\infty}$, and there are no other eigenvalues of $H_{\lambda}$ in $(a, b) .\left(\mathscr{E}_{\infty}\right.$ was defined in (H.3.4) to be $(a, b) \cap\left\{\right.$ point spectrum of $\left.H_{\infty}\right\}$.)

3. Given a specific eigenvalue trajectory $E_{m, \lambda}$, and given $\lambda_{1} \in P_{n}, \lambda_{2} \in P_{n+1}$, and $\lambda_{3} \in P_{n+2}$. By (2) $E_{m, \lambda_{1}}$ is within $\varepsilon$ of an element $E_{i}$ of $\mathscr{E}_{\infty}$. Let $E_{i+1}$ be the next lower element of $\mathscr{E}_{\infty}$, if it exists.

a. If $E_{i}$ is nondegenerate, and if $E_{i+1}$ exists, then $E_{m, \lambda_{2}}$ is within $\varepsilon$ of $E_{i+1}$. If $E_{i+1}$ does not exist, then $E_{m, \lambda_{2}}$ does not exist in $(a, b)$.

b. If $E_{i}$ is degenerate, and if $E_{i+1}$ exists, then either $E_{m, \lambda_{2}}$ or $E_{m, \lambda_{3}}$ is within $\varepsilon$ of $E_{i+1}$. If $E_{i+1}$ does not exist, then $E_{m, \lambda_{3}}$ does not exist in $(a, b)$.

With this definition in hand, we can state our results for the purely attractive potential. First we have the square-well-like case:

Theorem 5.2. Assume that $H_{\lambda}=-d^{2} / d x^{2}+V+\lambda W, \lambda>0$ satisfies (H.3.1), (H.3.2), and (H.3.4). Then the system exhibits the cascade phenomenon on the interval $(a, b)$ with $\delta=0$. The $n$-th transition region $T_{n}$ is centered at $\lambda=(n \pi / L)^{2}$, where $L=\int_{0}^{l} \sqrt{|W|} d x$. Moreover,

(i) On the plateau regions $\left(\lambda \in \bigcup_{n \in \mathbf{N}} P_{n}\right)$ the eigenvalues $E_{\lambda}$ of $H_{\lambda}$ are given by

$$
E_{\lambda}=E_{\infty}+\frac{c}{\sqrt{\lambda}} \cot \left(\sqrt{\lambda} L+O\left(\lambda^{-1 / 2}\right)\right)+O\left(\left(E_{\lambda}-E_{\infty}\right)^{2}\right)
$$

near each nondegenerate Dirichlet eigenvalue $E_{\infty}$, and by 


$$
\begin{aligned}
E_{\lambda}= & E_{\infty}+\frac{a}{\sqrt{\lambda}}\left\{\cot \left(\sqrt{\lambda} L+O\left(\lambda^{-1 / 2}\right)\right) \pm \sqrt{\cot ^{2}\left(\sqrt{\lambda} L+O\left(\lambda^{-1 / 2}\right)\right)+b}\right\} \\
& +O\left(\left(E_{\lambda}-E_{\infty}\right)^{2}\right)
\end{aligned}
$$

if $E_{\infty}$ is (twice) degenerate. Here $a, b$, and $c$ are positive parameters, independent of $\lambda$, that may depend on the eigenvalue $E_{\infty}$.

(ii) On the $n$-th transition region $T_{n}$ we have

$$
\lambda-\left(\frac{n \pi}{L}\right)^{2}=\frac{2}{L}\left(B_{-}(E)-B_{+}(E)-S(E)\right)+O\left(\lambda^{-1 / 2}\right),
$$

where the functions $B_{-}, B_{+}$, and $S$ are given below ((5.4) and (5.6)).

Remarks 5.3. Given (5.1), (5.2) and (5.3), the cascade phenomenon follows. Properties (1) and (2) are obvious. As for property (3), first suppose there are no degenerate Dirichlet eigenvalues. Equation (5.1) describes how, in the course of a plateau $P_{n}$, each eigenvalue trajectory $E_{\lambda}$ goes from slightly above a Dirichlet eigenvalue $E_{\infty}$ to slightly below $E_{\infty}$. At the beginning of $P_{n+1}, E_{\lambda}$ must be slightly above a (possibly different) Dirichlet eigenvalue (call it $E_{\infty}^{\prime}$ ), if it exists at all. By monotonicity, $E_{\infty}^{\prime}<E_{\infty}$, so in the transition region $T_{n} E_{\lambda}$ must drop from a neighborhood of $E_{\infty}$ to a neighborhood of $E_{\infty}^{\prime}$. This drop is described by (5.3). Since $B_{-}, B_{+}$, and $S$ are single-valued functions of energy, and since every eigenvalue trajectory $E_{\lambda}$ drops in $T_{n}, E_{\infty}^{\prime}$ must be the Dirichlet eigenvalue immediately below $E_{\infty}$. This establishes (3a). A similar argument involving degenerate eigenvalues and (5.2) establishes (3b).

Proof of Theorem 5.2. We use the notation of Theorem 3.3, letting $f_{ \pm}(E, x)$ be solutions of $\left(-d^{2} / d x^{2}+V\right) f_{ \pm}=E f_{ \pm}$in $L^{2}((l, \infty))$ and $L^{2}((-\infty, 0))$, respectively. We let $\mu=\sqrt{\lambda}$, we let $W_{0}, W_{l}, W_{0}^{\prime}$, and $W_{l}^{\prime}$ denote boundary values of $W$ and $W^{\prime}$ as in Sect. 3, and we define

$$
B_{-}(E)=\frac{1}{\sqrt{\left|W_{0}\right|}}\left(\frac{f^{\prime}(E, 0)}{f_{-}(E, 0)}+\frac{W_{0}^{\prime}}{4 W_{0}}\right) ; \quad B_{+}(E)=\frac{1}{\sqrt{\left|W_{l}\right|}}\left(\frac{f_{+}^{\prime}(E, l)}{f_{+}(E, l)}+\frac{W_{l}^{\prime}}{4 W_{l}}\right) .
$$

We shall construct an eigenfunction $\psi$ of $H_{\lambda}$. On $(-\infty, 0)$ and $(l, \infty) \psi$ must be a multiple of $f_{-}$and $f_{+}$, respectively. On $(0, l)$ we shall construct $\psi$ using the WKB approximation [22]. The matching conditions at 0 and $l$ will give (5.1), (5.2) and (5.3) near a nondegenerate eigenvalue of $H_{\infty}$, near a degenerate eigenvalue of $H_{\infty}$, and away from an eigenvalue of $H_{\infty}$, respectively.

On the interval $[0, l], \psi$ is a linear combination of

where

$$
\Phi_{ \pm}=\frac{\exp \left\{ \pm i \mu \int_{0}^{x} \sqrt{|W(x)|}\right\}}{\sqrt[4]{|W|}}\left\{1 \pm \frac{i}{\mu} \int_{0}^{x} a_{1}+O\left(\mu^{-2}\right)\right\},
$$

$$
a_{1}(E, x)=\frac{1}{\sqrt[4]{|W(x)|}}\left(E-V(x)+\frac{3}{16}\left(\frac{W^{\prime}(x)}{W(x)}\right)^{2}-\frac{W^{\prime \prime}(x)}{4 W(x)}\right) ; \quad S=\int_{0}^{l} a_{1}(E, x) d x .
$$


Taking a real trigonometric form of (5.6) we get

$$
\psi(E, x, \lambda)= \begin{cases}C_{-}(E, \lambda) f_{-}(E, x) & x<0 \\ \frac{A}{\sqrt[4]{|W(x)|}} \cos \left(\int_{0}^{x}\left(\mu \sqrt{\left|W\left(x^{\prime}\right)\right|}+\frac{a_{1}\left(E, x^{\prime}\right)}{\mu}+O\left(\mu^{-2}\right)\right) d x^{\prime}+\theta_{0}(E)\right) & 0<x<l, \\ C_{+}(E, \lambda) f_{+}(E, x) & x>l\end{cases}
$$

where the phase-shift $\theta_{0}(E)$ and the amplitudes $C_{ \pm}(E, \lambda)$ and $A(E, \lambda)$ are parameters, independent of $x$.

Matching $\psi^{\prime} / \psi$ at $x=0$ gives

$$
\mu \tan \theta_{0}=-B_{-}+O\left(\mu^{-2}\right)
$$

while matching at $x=l$ gives

$$
\mu \tan \left(\theta_{0}+\mu L+S / \mu+O\left(\mu^{-2}\right)\right)=-B_{+}+O\left(\mu^{-2}\right) .
$$

Combining these two conditions we get our desired matching condition

$$
\mu L+S / \mu \equiv \tan ^{-1}\left(B_{-} / \mu\right)-\tan ^{-1}\left(B_{+} / \mu\right)+O\left(\mu^{-2}\right) \quad(\bmod \pi),
$$

or equivalently

$$
\tan \left(\mu L+S / \mu+O\left(\mu^{-2}\right)\right)=\frac{B_{-} / \mu-B_{+} / \mu}{1+B_{-} B_{+} / \mu^{2}}
$$

Away from a Dirichlet Eigenvalue. The poles of $B_{ \pm}$occur precisely at the zeroes of $f_{+}(\cdot, l)$ and $f_{-}(\cdot, 0)$, i.e. at the eigenvalues of $H_{\infty}$. Away from these eigenvalues $B_{ \pm}$are bounded, so, for $\lambda$ large,

$$
\tan ^{-1}\left(B_{ \pm} / \mu\right)=B_{ \pm} / \mu+O\left(\mu^{-2}\right) .
$$

Thus $(5.10)$ reads

so

$$
\mu L+S / \mu=\left(B_{-}-B_{+}\right) / \mu+n \pi+O\left(\mu^{-2}\right)
$$

$$
\left(\mu-\frac{n \pi}{L}\right)=\frac{B_{-}-B_{+}-S}{\mu L}+O\left(\mu^{-2}\right) .
$$

Since $\lambda=\mu^{2}, d \lambda=2 \mu d \mu$, and (5.3) follows.

Near a Nondegenerate Dirichlet Eigenvalue. Let $E_{\infty}$ be a nondegenerate Dirichlet eigenvalue. Then either $f_{-}\left(E_{\infty}, 0\right)=0$ or $f_{+}\left(E_{\infty}, l\right)=0$, but not both (or else $E_{\infty}$ would be degenerate).

Suppose $f_{-}\left(E_{\infty}, 0\right)=0$. Since $f_{+}\left(E_{\infty}, l\right) \neq 0, B_{+}$is bounded in a neighborhood of $E_{\infty}$, so $\tan ^{-1}\left(B_{+} / \mu\right)$ is $O\left(\mu^{-1}\right)$, and (5.10) becomes

$$
\tan ^{-1}\left(B_{-} / \mu\right) \equiv \mu L+O\left(\mu^{-1}\right) \quad(\bmod \pi)
$$

or equivalently

$$
\frac{1}{B_{-}}=\mu^{-1} \cot \left(\mu L+O\left(\mu^{-1}\right)\right)
$$




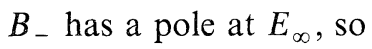

$$
\frac{1}{B_{-}}=c_{-} \Delta+O\left(\Delta^{2}\right)
$$

with $\Delta:=E_{\lambda}-E_{\infty}$ and $c_{-}=\sqrt{|W(l)|} d_{-}$, where $d_{-}$is the same positive constant that appeared in (3.27). As a result,

confirming (5.1).

$$
E_{\lambda}=E_{\infty}+\frac{1}{\mu c_{-}} \cot \left(\mu L+O\left(\mu^{-1}\right)\right)+O\left(\left(E_{\lambda}-E_{\infty}\right)^{2}\right),
$$

If instead $f_{+}\left(E_{\infty}, l\right)=0 \neq f_{-}\left(E_{\infty}, 0\right)$, a similar argument yields

$$
E_{\lambda}=E_{\infty}-\frac{1}{\mu c_{+}} \cot \left(\mu L+O\left(\mu^{-1}\right)\right)+O\left(\left(E_{\lambda}-E_{\infty}\right)^{2}\right),
$$

with $c_{+}<0$, again confirming (5.1).

Near a Degenerate Dirichlet Eigenvalue. At a degenerate Dirichlet eigenvalue both $B_{ \pm}$have poles, so we can write

$$
\frac{1}{B_{ \pm}}=c_{ \pm} \Delta+O\left(\Delta^{2}\right)
$$

Equation (5.11) then reads

$$
\tan \left(\mu L+O\left(\mu^{-1}\right)\right)=\frac{\left(c_{+}-c_{-}\right) \mu \Delta}{c_{+} c_{-} \mu^{2} \Delta^{2}+1}+\text { remainder terms }
$$

where the remainder terms either can be absorbed into the $O\left(\mu^{-1}\right)$ error in the phase, or contribute only $O\left(\Delta^{2}\right)$ to $E_{\lambda}$. Ignoring these terms gives a quadratic equation in $\mu \Delta$ :

$$
c_{+} c_{-}(\mu \Delta)^{2}+\left(c_{c}-c_{+}\right) \cot \left(\mu L+O\left(\mu^{-1}\right)\right)(\mu \Delta)+1=0
$$

with solution

$$
\begin{aligned}
\mu \Delta= & \frac{\left(c_{+}-c_{-}\right) \cot \left(\mu L+O\left(\mu^{-1}\right)\right) \pm \frac{\sqrt{\left(c_{+}-c_{-}\right)^{2} \cot ^{2}\left(\mu L+O\left(\mu^{-1}\right)\right)-4 c_{+} c_{-}}}{2 c_{+} c_{-}}}{} \\
& + \text {remainder terms. }
\end{aligned}
$$

Since $c_{+}<0<c_{-}$, and since the remainder terms will contribute only $\Delta^{2}$ to $E_{\lambda}$, we get (5.2), with

$$
a=\frac{c_{+}-c_{-}}{2 c_{+} c_{-}}>0 ; \quad b=\frac{-4 c_{+} c_{-}}{\left(c_{+}-c_{-}\right)^{2}}>0 .
$$

By Remark 5.3, this concludes the proof of Theorem 5.2.

Remark 5.4. The condition $\mu L \approx n \pi$, which marks the $n$-th transition region $T_{n}$, is a resonance condition which marks the threshold for the existence of the $n+1^{\text {st }}$ bound state of the well. If and only if this resonant condition is met, the wavefunction 
$\psi$ in the region $(0, l)$ is $O(1)$. Thus, by the Feynman-Hellman theorem [31]

$$
\frac{d E_{\lambda}}{d \lambda}=\langle\psi|W| \psi\rangle=\int_{0}^{l} W(x)|\psi(x)|^{2} d x
$$

$d E_{\lambda} / d \lambda$ is $O(1)$. If the resonant condition is not met, $\psi$ is $O\left(\lambda^{-1 / 2}\right)$ on $(0, l)$, and so $d E_{\lambda} / d \lambda$ is $O\left(\lambda^{-1}\right)$, as indicated in (5.1) and (5.2).

With a turning-point potential $W$ (H.3.3), the resonances are not as sharp as with a square-well-like potential (H.3.2). As a result the transition regions are wider $\left(O\left(\lambda^{1 / 6}\right)\right)$, and even away from the transition regions $E_{\lambda}-E_{\infty}$ is larger $\left(O\left(\lambda^{-1 / 3}\right)\right)$ than in the square-well-like case $\left(O\left(\lambda^{-1 / 2}\right)\right)$. Also, instead of encountering tangents and cotangents of $\mu L$, we see the trigonometric functions

$$
\Omega_{ \pm}(\alpha):=\frac{\cos (\alpha)}{\cos \left(\alpha \pm \frac{\pi}{3}\right)}=\frac{2 \cot (\alpha)}{\cot (\alpha) \mp \sqrt{3}}
$$

Theorem 5.5. Assume that $H_{\lambda}=-d^{2} / d x^{2}+V+\lambda W, \lambda>0$ satisfies (H.3.1), (H.3.3), and (H.3.4). Then the system exhibits the cascade phenomenon on the interval $(a, b)$ with $\delta=1 / 6$. Moreover,

(i) On the plateau regions the eigenvalues $E_{\lambda}$ of $H_{\lambda}$ are given by

$$
E_{\lambda}=E_{\infty}-c \lambda^{-1 / 3} \Omega_{+}\left(\sqrt{\lambda} L+O\left(\lambda^{-1 / 3}\right)\right)+O\left(\left(E_{\lambda}-E_{\infty}\right)^{2}\right)
$$

if $E_{\infty}$ is a nondegenerate Dirichlet eigenvalue, and

$$
\begin{aligned}
E_{\lambda}= & E_{\infty}-a \lambda^{-1 / 3}\left\{\Omega_{+}\left(\sqrt{\lambda} L+O\left(\lambda^{-1 / 3}\right)\right)\right. \\
& \pm \sqrt{\left.\Omega_{+}^{2}\left(\sqrt{\lambda} L+O\left(\lambda^{-1 / 3}\right)\right)+b \frac{\Omega_{+}\left(\sqrt{\lambda} L+O\left(\lambda^{-1 / 3}\right)\right)}{\Omega_{-}\left(\sqrt{\lambda} L+O\left(\lambda^{-1 / 3}\right)\right)}\right\}} \\
& +O\left(\left(E_{\lambda}-E_{\infty}\right)^{2}\right)
\end{aligned}
$$

if $E_{\infty}$ is (twice) degenerate. Here $a, b$, and $c$ are positive parameters, independent of $\lambda$, that may depend on the eigenvalue $E_{\infty}$, and $L=\int_{0}^{l} \sqrt{|W|}$, as in Theorem 5.2.

(ii) The $n$-th transition region $T_{n}$ is centered at $\lambda=\left(\left(n+\frac{1}{6}\right) \pi / L\right)^{2}$, and has width $O\left(\lambda^{1 / 6}\right)$.

Proof. The proof follows the pattern of the proof of Theorem 5.2, and we use much the same notation. $f_{ \pm}(E, x), \mu, \Delta, W_{0}^{\prime}$, and $W_{l}^{\prime}$ are as in Theorem 5.2. Our boundary data $B_{ \pm}(E)$ are now defined as

$$
B_{-}(E)=\sqrt[3]{\left|W_{0}^{\prime}\right|}\left(\frac{f_{-}\left(E, 0_{-}\right)}{f_{-}^{\prime}\left(E, 0_{-}\right)}\right) ; \quad B_{+}(E)=\sqrt[3]{W_{l}^{\prime}}\left(\frac{f_{+}\left(E, l_{+}\right)}{f_{+}^{\prime}\left(E, l_{+}\right)}\right) .
$$

(Note that these functions have zeroes rather than poles at the Dirichlet eigenvalues $E_{\infty}$.)

As with Theorem 5.2, we construct an eigenfunction $\psi$ of $H_{\lambda}$. The wavefunction 
must be a multiple of $f_{-}$and $f_{+}$on $(-\infty, 0)$ and $(l, \infty)$, respectively. On $(0, l)$ we again use the WKB approximation [22], but (5.6) is no longer valid near the turning points 0 and $l$. Near those points we must interpolate with Airy functions, as in Theorem 3.3. Our wavefunction on $(0, l)$ is

$$
\Phi_{\mathrm{WKB}}= \begin{cases}p-\mathrm{Ai}\left(-\left|\lambda W_{0}^{\prime}\right|^{1 / 3} x\right)+q_{-} \mathrm{Bi}\left(-\left|\lambda W_{0}^{\prime}\right|^{1 / 3} x\right)+O\left(\lambda^{-1 / 3}\right) & \text { for } x \text { near } 0 \\ A|W(x)|^{-1 / 4} \cos \left(\int_{0}^{x} \mu \sqrt{|W|}+O\left(\mu^{-1}\right)+\frac{\pi}{4}+\theta_{0}\right)\left(1+O\left(\mu^{-1}\right)\right) & x \text { away from } 0, l, \\ p_{+} \operatorname{Ai}\left(-\left|\lambda W_{l}^{\prime}\right|^{1 / 3}(l-x)\right)+q_{+} \mathrm{Bi}\left(-\left|\lambda W_{l}^{\prime}\right|^{1 / 3}(l-x)\right)+O\left(\lambda^{-1 / 3}\right) & \text { for } x \text { near } l\end{cases}
$$

where $p_{ \pm}, q_{ \pm}$, and $A$ are constants.

We recall some facts about Airy functions [22]. For large negative arguments, the Airy functions take the asymptotic form

$$
\begin{aligned}
& \operatorname{Ai}(-z)=\frac{z^{-1 / 4}}{\sqrt{\pi}}\left(\sin \left(\xi+\frac{\pi}{4}\right)+O\left(\xi^{-1}\right)\right), \\
& \operatorname{Bi}(-z)=\frac{z^{-1 / 4}}{\sqrt{\pi}}\left(\cos \left(\xi+\frac{\pi}{4}\right)+O\left(\xi^{-1}\right)\right),
\end{aligned}
$$

where $\xi=2 z^{3 / 2} / 3, z>0$. The behavior of these functions near zero is given by the Wronskian matrix

$$
\left(\begin{array}{ll}
\mathrm{Ai}(0) & \mathrm{Bi}(0) \\
\mathrm{Ai}^{\prime}(0) & \mathrm{Bi}^{\prime}(0)
\end{array}\right)=\left(\begin{array}{ll}
1 /\left\{3^{2 / 3} \Gamma(2 / 3)\right\} & 1 /\left\{3^{1 / 6} \Gamma(2 / 3)\right\} \\
-1 /\left\{3^{1 / 3} \Gamma(1 / 3)\right\} & 3^{1 / 6} /\{\Gamma(2 / 3)\}
\end{array}\right) .
$$

To relate $\theta_{0}$ to $B_{-}$, we must first relate $\theta_{0}$ to $p_{-} / q_{-}$, and then relate $p_{-} / q_{-}$to $B_{\text {. }}$. The first part is easy, given (5.31).

$$
p_{-} / q_{-}=-\tan \left(\theta_{0}\right) .
$$

Relating $p_{-} / q_{-}$to $B_{-}$is a bit trickier. By (5.30) we have

so

$$
\left(\begin{array}{c}
f_{-}(0) \\
f_{-}^{\prime}(0)
\end{array}\right)=\left(\begin{array}{cc}
1 & 0 \\
0 & -\left|\lambda W_{0}^{\prime}\right|^{1 / 3}
\end{array}\right)\left(\begin{array}{cc}
\mathrm{Ai}(0) & \mathrm{Bi}(0) \\
\mathrm{Ai}^{\prime}(0) & \mathrm{Bi}^{\prime}(0)
\end{array}\right)\left(\begin{array}{c}
p_{-} \\
q_{-}
\end{array}\right)
$$

$$
B_{-}(E)=\omega \lambda^{-1 / 3} \frac{\tan \left(\theta_{0}+O\left(\lambda^{-1 / 3}\right)\right)-\sqrt{3}}{\tan \left(\theta_{0}+O\left(\lambda^{-1 / 3}\right)\right)+\sqrt{3}}=\omega \lambda^{-1 / 3} \frac{\sin \left(\theta_{0}-\pi / 3+O\left(\lambda^{-1 / 3}\right)\right)}{\sin \left(\theta_{0}+\pi / 3+O\left(\lambda^{-1 / 3}\right)\right)},
$$

where $\omega=3^{-1 / 3} \Gamma\left(\frac{1}{3}\right) / \Gamma\left(\frac{2}{3}\right)$. This may also be inverted to give

$$
\tan \left(\theta_{0}+O\left(\lambda^{-1 / 3}\right)\right)=\sqrt{3}\left(\frac{\omega+B_{-} \lambda^{1 / 3}}{\omega-B_{-} \lambda^{1 / 3}}\right) .
$$

If we define $\theta_{l}:=\mu L+\pi / 2+\theta_{0}$, a similar calculation near $x=l$ yields

$$
B_{+}(E)=-\omega \lambda^{-1 / 3} \frac{\tan \left(\theta_{l}+O\left(\lambda^{-1 / 3}\right)\right)+\sqrt{3}}{\tan \left(\theta_{l}+O\left(\lambda^{-1 / 3}\right)\right)-\sqrt{3}},
$$


or equivalently,

$$
\tan \left(\theta_{l}+O\left(\lambda^{-1 / 3}\right)\right)=-\sqrt{3}\left(\frac{\omega-B_{+} \lambda^{1 / 3}}{\omega+B_{+} \lambda^{1 / 3}}\right) .
$$

All that remains is to analyze these expressions in the various regimes.

Away from a Dirichlet Eigenvalue. $B_{ \pm}$are both bounded away from zero, so by (5.36) and (5.38) we have

$$
\theta_{0 / l}=\tan ^{-1}\left(\mp \sqrt{3}+O\left(\lambda^{-1 / 3}\right)\right)+O\left(\lambda^{-1 / 3}\right) \equiv \mp \frac{\pi}{3}+O\left(\lambda^{-1 / 3}\right) \quad(\bmod \pi),
$$

and so

$$
\mu L=\theta_{l}-\theta_{0}-\frac{\pi}{2}=\left(n+\frac{1}{6}\right) \pi+O\left(\lambda^{-1 / 3}\right)
$$

and

$$
\lambda=\left(\frac{\left(n+\frac{1}{6}\right) \pi}{L}\right)^{2}+O\left(\lambda^{1 / 6}\right) .
$$

This establishes the location and width of the transition regions.

Near a Nondegenerate Dirichlet Eigenvalue. Let $E_{\infty}$ by a nondegenerate Dirichlet eigenvalue with $B_{-}\left(E_{\infty}\right)=0$. Since $B_{+}(E)$ is bounded away from zero for $E$ near $E_{\infty}$, we have $\theta_{l}=\left(n+\frac{1}{3}\right) \pi+O\left(\lambda^{-1 / 3}\right)$. Thus $\theta_{0}=-\mu L-\pi / 6+O\left(\lambda^{-1 / 3}\right)$ and

$$
B_{-}(E)=\omega \lambda^{-1 / 3} \frac{\sin \left(\mu L+\pi / 2+O\left(\lambda^{-1 / 3}\right)\right)}{\sin \left(\mu L-\pi / 6+O\left(\lambda^{-1 / 3}\right)\right)}=-\omega \lambda^{-1 / 3} \Omega_{+}\left(\mu L+O\left(\lambda^{-1 / 3}\right)\right) .
$$

Since $B_{-}(E)$ has a zero at $E_{\infty}$ we can write

$$
B_{-}(E)=c_{-} \Delta+O\left(\Delta^{2}\right)
$$

Since $c_{-}>0$ we then have

$$
E_{\lambda}=E_{\infty}-\frac{\omega}{c_{-}} \lambda^{-1 / 3} \Omega_{+}\left(\mu L+O\left(\lambda^{-1 / 3}\right)\right)
$$

confirming (5.27).

Near a nondegenerate Dirichlet eigenvalue with $B_{+}\left(E_{\infty}\right)=0$, a similar argument yields

$$
E_{\lambda}=E_{\infty}+\frac{\omega}{c_{+}} \lambda^{-1 / 3} \Omega_{+}\left(\mu L+O\left(\lambda^{-1 / 3}\right)\right),
$$

with $c_{+}<0$, again confirming (5.27).

Near a Degenerate Dirichlet Eigenvalue. We note that

$$
\tan \left(\mu L+\frac{\pi}{2}+O\left(\hat{\lambda}^{-1 / 3}\right)\right)=\tan \left(\theta_{l}-\theta_{0}\right)=\frac{\tan \left(\theta_{l}\right)-\tan \left(\theta_{0}\right)}{1+\tan \left(\theta_{l}\right) \tan \left(\theta_{0}\right)} .
$$


By (5.36) and (5.38) this becomes

$$
\begin{aligned}
& \tan \left(\mu L+\frac{\pi}{2}+O\left(\lambda^{-1 / 3}\right)\right) \\
& =-\sqrt{3}\left(\frac{\left(\omega-B_{+} \lambda^{1 / 3}\right)\left(\omega-B_{-} \lambda^{1 / 3}\right)+\left(\omega+B_{+} \lambda^{1 / 3}\right)\left(\omega+B_{-} \lambda^{1 / 3}\right)}{\left(\omega+B_{+} \lambda^{1 / 3}\right)\left(\omega-B_{-} \lambda^{1 / 3}\right)-3\left(\omega-B_{+} \lambda^{1 / 3}\right)\left(\omega+B_{-} \lambda^{1 / 3}\right)}\right) .
\end{aligned}
$$

Defining $Z:=-\tan \left(\mu L+\pi / 2+O\left(\lambda^{-1 / 3}\right)\right)=\cot \left(\mu L+O\left(\lambda^{-1 / 3}\right)\right)$ and eliminating the denominator, this becomes

$$
B_{+} B_{-} \lambda^{2 / 3}(Z-\sqrt{3})+2 \omega\left(B_{+}-B_{-}\right) \lambda^{1 / 3}-\omega^{2}(Z+\sqrt{3})=0 .
$$

At a degenerate Dirichlet eigenvalue both $B_{ \pm}=0$, so we can write

$$
B_{ \pm}(E)=c_{ \pm} \Delta+O\left(\Delta^{2}\right) .
$$

Plugging this into (5.48) gives a quadratic equation in $\left(\lambda^{1 / 3} \Delta\right)$ :

$$
\begin{aligned}
& c_{+} c_{-}(Z-\sqrt{3})\left(\lambda^{1 / 3} \Delta\right)^{2}+2 \omega Z\left(c_{+}-c_{-}\right)\left(\lambda^{1 / 3} \Delta\right)-\omega^{2}(Z+\sqrt{3}) \\
& \quad+\text { remainder terms }=0,
\end{aligned}
$$

with solution

$$
\lambda^{1 / 3} \Delta=\omega \frac{-Z\left(c_{+}-c_{-}\right) \pm \sqrt{\left(c_{+}-c_{-}\right)^{2} Z^{2}+c_{+} c_{-}\left(Z^{2}-3\right)}}{c_{+} c_{-}(Z-\sqrt{3})}+\text { remainder terms }
$$

Since $c_{+}<0<c_{-}$, since $Z /(Z \pm \sqrt{3})=\frac{1}{2} \Omega_{\mp}\left(\mu L+O\left(\lambda^{-1 / 3}\right)\right)$, and since the remainder terms only contribute $O\left(\Delta^{2}\right)$ to $E_{\lambda}$, this gives (5.28) with

$$
a=\frac{\omega\left(c_{+}-c_{-}\right)}{2 c_{+} c_{-}}>0 ; \quad b=\frac{-c_{+} c_{-}}{\left(c_{+}-c_{-}\right)^{2}}>0
$$

This concludes the proof of Theorem 5.5.

Remark 5.6. The formation of plateaus and transition regions in Theorems 5.2 and 5.5 is independent of the particular shape of the background potential $V$. $V$ merely determines the Dirichlet eigenvalues $E_{\infty}$ and the constants $c_{ \pm} . W$, on the other hand, determines the location and size of the transition regions, and the shape of the eigenvalue trajectories. In particular, if the support of $W$ shrinks to a point and $W$ is replaced by a $\delta$-function, the cascade phenomenon disappears, and the spectrum of $H_{\lambda}$ approaches that of $H_{\infty}$ as $\lambda \rightarrow \pm \infty$. We study an analytically solvable example in Sect. 7.

Remark 5.7. The condition for being in a transition region $(\mu L=n \pi$ for squarewell-like potentials $W$ or $\mu L=\left(n+\frac{1}{6}\right) \pi$ for turning-point potentials) is the same as the condition for the existence of threshold (zero energy) resonances of $-d^{2} / d x^{2}+\lambda W$. The well known fact that square-well-like potentials produce more pronounced resonances than turning-point potentials accounts for the wider transition regions and slower approach to $E_{\infty}$ in Theorem 5.5 compared to 5.2. 
Remark 5.8. The cascade phenomenon for square-well-like potentials was first observed by Zel'dovich [36] (see also [11,21, 23, 24,33]). He studied the system

$$
V(x)=\left\{\begin{array}{cc}
0 & r \leqq d \\
-r^{-1} & r>d
\end{array}, \quad W(x)=\left\{\begin{array}{rl}
-1 & r \leqq d \\
0 & r>d
\end{array},\right.\right.
$$

$d>0$, on the half-line $(0, \infty)$. This case is covered by our methods by simply demanding $h_{-}(E) \equiv 0, E \in \mathbf{R}$. (Indeed, choosing appropriate boundary conditions at $r=0_{+}$[6], our formalism easily generalizes to spherically symmetric potentials in $v \geqq 2$ dimensions.) While Zel'dovich exhibits the resonance behavior mentioned above, he is somewhat vague about the significance of the Dirichlet boundary condition at $r=d$. Since he is merely interested in the case $d \ll 1$, he essentially replaces $H_{\infty}$ with the Coulomb Hamiltonian on $(0, \infty)$, and identifies the asymptotic eigenvalues as Coulomb levels.

\section{The Cascade Phenomenon for $W$ 's of Indefinite Sign}

This section extends the results of Sect. 5, in that we now consider perturbations $W$ which change sign. For such a $W$, we call the intervals where $W<0$ "wells," and the intervals where $W>0$ "barriers." The goal of this section is to show that all perturbations $W$ that change sign give rise to the cascade phenomenon of Sect. 5 .

To treat these more complicated functions, we introduce additional hypotheses.

For the square-well-like perturbations, we require:

(H.6.1) $W$ is a function, supported on $[0, l]$, that is $C^{2}$ except at $0, l$, and a finite number of points $\left\{p_{1}, \ldots, p_{n}\right\}$, where it may have a jump discontinuity. $W$ is bounded away from zero on $(0, l)$, and is not positive semi-definite (i.e. $|W(x)|>\varepsilon>0$ for all $x \in(0, l)$, and $W(x)<0$ for some $\left.x \in(0, l) \backslash\left\{p_{1}, \ldots, p_{2}\right\}\right)$.

In the turning-point case, we want a function, supported on $[0, l]$, that oscillates, but with only simple zeroes. To get this, we introduce

$$
W(x)=\left\{\begin{array}{ll}
0 & x<0 \text { or } x>l \\
\tilde{W}(x) & 0 \leqq x \leqq l
\end{array},\right.
$$

where $\tilde{W}(x)$ is a $C^{2}$ function with no multiple zeroes, and with simple zeroes at 0 and $l$ (and perhaps elsewhere). We also assume that $W(x)<0$ for some $x \in(0, l)$.

Notice that hypotheses (H.3.2) and (H.3.3) are special cases of (H.6.1) and (H.6.2), respectively.

We also introduce a special case, shown in Fig. 6.1, which we shall study in detail. This case is covered by the hypothesis.

(H.6.3) $W(x)$ satisfies (H.6.2), with the zeroes of $\tilde{W}$ coming at $0, \alpha, \beta, \gamma$, and $l$, with $0<\alpha<\beta<\gamma<l$, and with $W(x)<0$ for all $x \in(0, \alpha)$.

In each of these cases, we define $H_{0}, H_{\lambda}$ in $L^{2}(\mathbf{R})$ and $H_{\infty}$ in $L^{2}(\mathbf{R} \backslash \Sigma)$ as before. We consider $\lambda>0$, and define $\mu=\sqrt{\lambda}$. Our main result is 


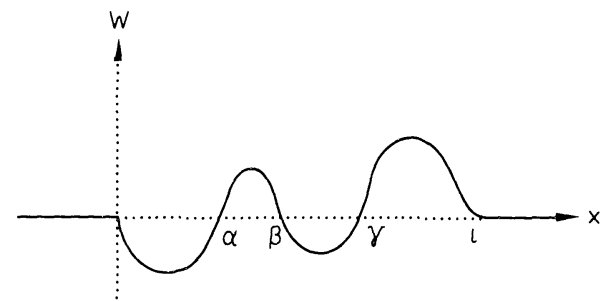

Fig. 6.1. A mixed-sign potential

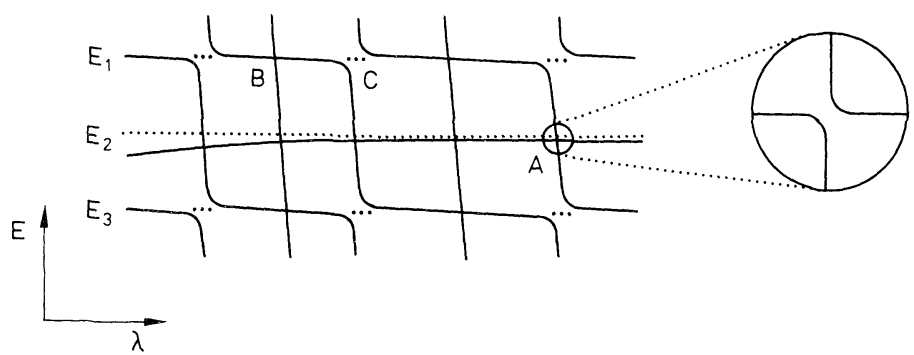

Fig. 6.2. The casecade phenomenon with a mixed-sign potential

Theorem 6.1. Let $H_{\lambda}=-d^{2} / d x^{2}+V+\lambda W, \lambda>0$ satisfy $(H .3 .1),(H .3 .4)$ and either (H.6.1) or (H.6.2). The system then exhibits the cascade phenomenon on the interval $(a, b)$. Moreover,

(i) If (H.6.1) is true, then the size of the transition regions $T_{n}$ remains $O(1)$ as $\lambda \rightarrow \infty$. If (H.6.2) is true, some of the transition regions may grow as $\lambda^{1 / 6}$.

(ii) If there is an exterior barrier on the left-hand side (i.e. if $\lim \operatorname{sgn}(W(x))=1$ ), then, for all $\lambda$ sufficiently large, there is at least one eigenvalue of $H_{\lambda}$ within $\varepsilon$ of each eigenvalue of $H_{-}$in $(a, b)$. That is, in each transition region, as one eigenvalue trajectory approaches the neighborhood of a Dirichlet eigenvalue $E_{\infty}$ and another departs, the arrival occurs before the departure. (This is shown in Fig. 6.2, near the Dirichlet eigenvalue $E_{2}$.)

(iii) If there is an exterior barrier on the right-hand side (i.e. if $\lim _{x \rightarrow l-} \operatorname{sgn}(W(x))=1$ ), then a similar result applies at the eigenvalues of $H_{+}$.

(iv) If there are exterior barriers on both sides, then for all $\lambda$ sufficiently large, there are at least two eigenvalues of $H_{\lambda}$ within $\varepsilon$ of each degenerate eigenvalue of $H_{\infty}$.

Remark 6.2. The cascade phenomenon requires that eigenvalue trajectories descend from Dirichlet eigenvalue to Dirichlet eigenvalue. Unlike the purely attractive case, however, this descent need not be monotonic. On some plateaus $d E_{\lambda} / d \lambda$ may be positive, as in Fig. 6.2.

Remark 6.3. If the perturbation $W$ contains $N$ wells, then there exist periods $L_{i}$ and phase-shifts $\phi_{i},(i=1, \ldots, N)$, such that each well contributes its own transition regions $T_{n}^{(i)}$, located near the points $\lambda=\left(n \pi+\phi_{i}\right)^{2} / L_{i}^{2},(n=1,2, \ldots)$, independent 
of the other wells. The widths of the transition regions corresponding to interior wells remain $O(1)$ as $\lambda \rightarrow \infty$, but the transition regions corresponding to exterior wells may grow as $\lambda^{1 / 6}$ if (H.6.2) holds.

Remark 6.4. The assumptions of Theorem 6.1 may be relaxed somewhat, in that we may allow functions $W$ that have both square-well-like and turning-point zero-crossings (e.g. Fig. 6.3). In such cases, the size of the transitions regions $T_{n}$ remain $O(1)$ if the crossings at 0 and $l$ are square-well-like; otherwise, some transition regions may grow as $\lambda^{1 / 6}$.

The proof of this theorem involves considering a large number of cases. The arguments are essentially the same for all cases, but the calculations differ somewhat. We will prove the specific case (H.6.3) in detail, and then explain how to handle other cases.

Proof of case (H.6.3). We refer to the intervals $(0, \alpha),(\alpha, \beta),(\beta, \gamma)$, and $(\gamma, l)$ as "well 1," "barrier 2," "well 3," and "barrier 4," respectively. We define the periods

$$
L_{1} \equiv \int_{0}^{\alpha} \sqrt{|W(x)|} d x ; \quad L_{2} \equiv \int_{\alpha}^{\beta} \sqrt{W(x)} d x ; \quad L_{3} \equiv \int_{\beta}^{\gamma} \sqrt{|W(x)|} d x ; \quad L_{4} \equiv \int_{\gamma}^{l} \sqrt{W(x)} d x
$$

and the boundary values

$$
W_{p}^{\prime}=d W /\left.d x\right|_{x=p} ; \quad p \in\{0, \alpha, \beta, \gamma, l\} .
$$

We also define the ratios

$$
h_{p}(E, \lambda) \equiv \psi(p) / \psi^{\prime}(p)
$$

For $p \in\{0, l\}$ we have

$$
h_{0}(E)=f_{-}(E, 0) / f_{-}^{\prime}(E, 0) ; \quad h_{l}(E)=f_{+}(E, l) / f_{+}^{\prime}(E, l),
$$

which depends on external data, and so are functions of $E$ only.

Finally, we note that in each barrier there are two linearly independent solutions to the Schrödinger equation, one exponentially increasing and one exponentially decreasing. Let $\Phi_{+(-)}^{(i)}$ be the increasing (decreasing) solution in barrier \#i. Define

$$
\begin{array}{ll}
g_{\alpha}=\Phi_{-}^{(2)}(\alpha) / \Phi_{-}^{(2)^{\prime}}(\alpha), & g_{\beta}=\Phi_{+}^{(2)}(\beta) / \Phi_{+}^{(2)^{\prime}}(\beta), \\
g_{\gamma}=\Phi_{-}^{(4)}(\gamma) / \Phi_{-}^{(4)^{\prime}}(\gamma), & g_{l}=\Phi_{+}^{(4)}(l) / \Phi_{+}^{(4)^{\prime}}(l) .
\end{array}
$$

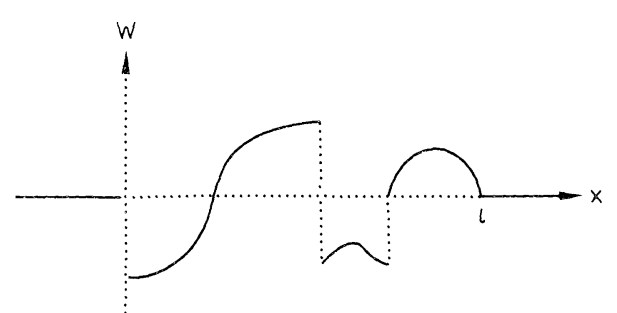

Fig. 6.3. A more general potential $W$ 
From the analysis of Sect. 5 we know that

$$
\begin{array}{ll}
g_{\alpha}=-\omega\left(W_{\alpha}^{\prime} \lambda\right)^{-1 / 3}+O\left(\lambda^{-2 / 3}\right), & g_{\beta}=\omega\left|W_{\beta}^{\prime} \lambda\right|^{-1 / 3}+O\left(\lambda^{-2 / 3}\right), \\
g_{\gamma}=-\omega\left(W_{\gamma}^{\prime} \lambda\right)^{-1 / 3}+O\left(\lambda^{-2 / 3}\right), & g_{l}=\omega\left|W_{l}^{\prime} \lambda\right|^{-1 / 3}+O\left(\lambda^{-2 / 3}\right),
\end{array}
$$

where $\omega=3^{-1 / 3} \Gamma(1 / 3) / \Gamma(2 / 3)$.

Solving the Schrödinger equation on the interval $(0, \alpha)$ provides a matching condition for $h_{0}$ and $h_{\alpha}$. Similarly, solving the Schrödinger equation on the interval $(\alpha, \beta)$ provides a matching condition for $h_{\alpha}$ and $h_{\beta}$, and so on. (These calculations were essentially done in the proofs of Theorems 3.3 (for the barriers) and 5.2 (for the wells), and so need not be repeated.

For the barriers we have that

and that

$$
\left(h_{\alpha}-g_{\alpha}\right)\left(h_{\beta}-g_{\beta}\right)=O\left(e^{-2 \mu L_{2}}\right),
$$

$$
\left(h_{\gamma}-g_{\gamma}\right)\left(h_{l}-g_{l}\right)=O\left(e^{-2 \mu L_{4}}\right) .
$$

As a result we have that either

or

$$
h_{\alpha}=g_{\alpha}+O\left(e^{-\mu L_{2}}\right)
$$

and that either

$$
h_{\beta}=g_{\beta}+O\left(e^{-\mu L_{2}}\right)
$$

$$
h_{\gamma}=g_{\gamma}+O\left(e^{-\mu L_{4}}\right)
$$

or

$$
h_{l}=g_{l}+O\left(e^{-\mu L_{4}}\right) \text {. }
$$

For the wells we have that

$$
\theta_{\alpha}-\theta_{0}=\mu L_{1}+\frac{\pi}{2}+O\left(\lambda^{-1 / 3}\right)
$$

and

$$
\theta_{\gamma}-\theta_{b}=\mu L_{3}+\frac{\pi}{2}+O\left(\lambda^{-1 / 3}\right)
$$

where

$$
\begin{array}{ll}
\tan \theta_{0}=\sqrt{3}\left(\frac{\omega+h_{0}\left|\lambda W_{0}^{\prime}\right|^{1 / 3}}{\omega-h_{0}\left|\lambda W_{0}^{\prime}\right|^{1 / 3}}\right), & \tan \theta_{\alpha}=-\sqrt{3}\left(\frac{\omega-h_{\alpha}\left|\lambda W_{\alpha}^{\prime}\right|^{1 / 3}}{\omega+h_{\alpha}\left|\lambda W_{\alpha}^{\prime}\right|^{1 / 3}}\right), \\
\tan \theta_{\beta}=\sqrt{3}\left(\frac{\omega+h_{\beta}\left|\lambda W_{0}^{\prime}\right|^{1 / 3}}{\omega-h_{\beta}\left|\lambda W_{0}^{\prime}\right|^{1 / 3}}\right), & \tan \theta_{\gamma}=-\sqrt{3}\left(\frac{\omega-h_{\gamma}\left|\lambda W_{\alpha}^{\prime}\right|^{1 / 3}}{\omega+h_{\gamma}\left|\lambda W_{\alpha}^{\prime}\right|^{1 / 3}}\right) .
\end{array}
$$

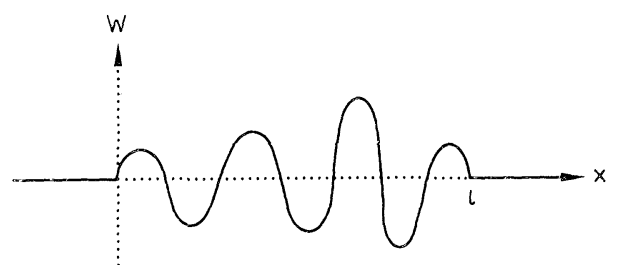

Fig, 6.4. A potential with several interior wells 
Equation (6.9b) means that, unless $\mu \approx\left(n+\frac{1}{2}\right) \pi / L_{3}$, Eqs. (6.8b) and (6.8c) cannot both hold. Therefore, either (6.8d) holds, or $(6.8 \mathrm{a})$ holds, or $\mu \approx\left(n+\frac{1}{2}\right) \pi / L_{3}$. We examine these possibilities, one at a time.

(Notice that Eqs. (6.8) and (6.9) apply a condition on $h_{l}$ or a condition on $h_{\alpha}$ (and hence on $h_{0}$ via Eq. (6.9a)), or on $L_{3}$, but not on any two together. The repulsive potentials effectively decouple the right-hand, left-hand, and interior problems. In a more complicated case, e.g., that shown in Fig. 6.4, we would have a right-hand problem, a left-hand problem, and one problem for each of the interior wells, all decoupled. A solution to the whole problem would be a solution to any one of these sub-problems.)

If Eq. (6.8d) holds, we proceed as in the proof of Theorem 3.3. E must be close to an eigenvalue $E_{\infty,+}$ of $H_{+}$, so

and so

$$
h_{l}(E)=d_{l}\left(E-E_{\infty,+}\right)+O\left(\left(E-E_{\infty,+}\right)^{2}\right),
$$

$$
E_{\lambda}=E_{\infty,+}+\frac{\omega}{d_{l}}\left|\lambda W_{l}^{\prime}\right|^{-1 / 3}+O\left(\lambda^{-2 / 3}\right) .
$$

This provides the plateaus near the eigenvalues of $H_{+}$. Since $d_{l}<0$, in these plateaus $E_{\lambda}$ is increasing, not decreasing.

If instead Eq. (6.8a) holds, we proceed as in the proof of Theorem 5.5. From (6.10a) we have $\theta_{\alpha}=\left(n+\frac{1}{2}\right) \pi$, so $\theta_{0}=n \pi-\mu L_{1}$. By (5.35) we have

$$
h_{0}(E)=\omega\left|\lambda W_{0}^{\prime}\right|^{-1 / 3} \frac{\tan \left(\mu L_{1}+O\left(\lambda^{-1 / 3}\right)\right)+\sqrt{3}}{\tan \left(\mu L_{1}+O\left(\lambda^{-1 / 3}\right)\right)-\sqrt{3}} .
$$

Near an eigenvalue $E_{\infty,-}$ of $H_{-}$,

$$
h_{0}(E)=d_{0}\left(E-E_{\infty,-}\right)+O\left(\left(E-E_{\infty,-}\right)^{2}\right),
$$

so

$$
E_{\lambda}=E_{\infty,-}+\frac{\omega}{d_{0}}\left|\lambda W_{0}^{\prime}\right|^{-1 / 3} \frac{\tan \left(\mu L_{1}+O\left(\lambda^{-1 / 3}\right)\right)+\sqrt{3}}{\tan \left(\mu L_{1}+O\left(\lambda^{-1 / 3}\right)\right)-\sqrt{3}} .
$$

This provides the plateaus near the eigenvalues of $H_{-}$as well as describing some transition regions, which occur when $\mu L_{1} \approx\left(n+\frac{1}{3}\right) \pi$. The size of the transition regions may be calculated precisely as in the proof of Theorem 5.5.

Finally, there is the possibility that (6.8b) and (6.8c) both occur. In this case both $\theta_{\beta}$ and $\theta_{\gamma}$ are congruent to $\pi / 2(\bmod \pi)$, giving us a condition,

$$
\mu L_{3}=\left(n+\frac{1}{2}\right) \pi+O\left(\lambda^{-1 / 3}\right),
$$

that is utterly insensitive to external data. Equation (6.16) describes a whole new set of transition regions. The location of these regions is only specified to $O\left(\lambda^{1 / 6}\right)$. However, the width of these regions is $O(1)$. (The reason is that (6.16) essentially describes bound states of well 3 , with $\psi$ large in well 3 and decaying exponentially in barriers 2 and 4. The Feynman-Hellman theorem [31] then implies that $d E_{\lambda} / d \lambda$ is negative and $O(1)$, so the width of these regions must be $O(1)$.)

We now have equations for all three types of solutions; namely, the solutions 
to the right-hand problem (Eq. (6.12)), the solutions to the left-hand problem (Eq. (6.15)), and the solutions to the interior problem (Eq. (6.16)). To get a complete picture, we merely include all three types.

The generic situation is shown in Fig. 6.2, where $E_{1}$ and $E_{3}$ are eigenvalues of $E_{\infty,-}$, and $E_{2}$ is an eigenvalue of $H_{+}$. Because of the no-crossing rule, a given eigenvalue trajectory cannot go directly from $E_{1}$ to $E_{3}$, but must first stop at $E_{2}$. Solutions to Eq. (6.16) appear as nearly vertical lines. By the no-crossing rule, neither these (nearly) vertical lines nor the nearly horizontal lines coming from Eq. (6.12) describe single trajectories; each describes a series of curves which are separated by exponentially small gaps, as at point $A$ in the figure. Each eigenvalue trajectory is, at various points, described by Eq. (6.12), (6.15), and (6.16).

A (twice) degenerate eigenvalue $E_{\infty,+}$ of $H_{\infty}$ is an eigenvalue of both the right and left Dirichlet operators $H_{+}$and $H_{-}$. Near such an energy, Eqs. (6.8a) and (6.8d) may both be satisfied. This gives rise to two eigenvalues of $H_{\lambda}$, one given by Eq. (6.12) and one given by Eq. (6.15). At a transition region, only the lower of the two trajectories may leave the vicinity of $E_{\infty,+}$, because of the no-crossing rule. As a result, each trajectory stays near the Dirichlet eigenvalue for the length of two plateaus, as required.

In short, away from the transition regions $T_{n}^{(1)}$ given by $\lambda=\left(n+\frac{1}{3}\right)^{2} \pi^{2} / L_{1}^{2}+$ $O\left(\lambda^{1 / 6}\right)$, and away from the transition regions $T_{n}^{(2)}$ given by $\lambda=\left(n+\frac{1}{2}\right)^{2} \pi^{2} / L_{3}^{2}+O(1)$, the spectrum of $H_{\lambda}$ is essentially that of $H_{\infty}$. At each transition region the spectrum shifts down by one. Near the eigenvalues of $H_{+}$, the eigenvalue trajectories come exponentially close to crossing (e.g. at point $A$ in Fig. 6.2), so there is always at least one trajectory within $\varepsilon$ of $E_{\infty,+}$. (There are near-crossings elsewhere, e.g. at

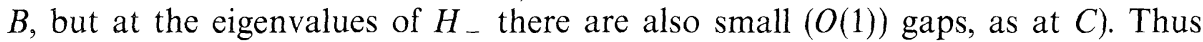
the conclusions of Theorem 6.1 are satisfied in the special case (H.6.3).

Remark 6.5. The potentials that satisfy (H.6.1) or (H.6.2) can be divided into four classes. First come the potentials with barriers at both ends, and with one or more wells in between (e.g. Fig. 6.5a). Next come the potentials with a barrier at one end, a well at the other end, and possibly some interior wells (e.g. Fig. 6.5b). Third come the potentials with wells at both ends, and possibly some interior wells (e.g. Fig. 6.5c). The final class, covered in Sect. 5, consists of the purely attractive potentials, where a single well governs both the right- and left-hand problems. The qualitative dependence of $E_{\lambda}$ on $\lambda$ for the three classes is shown in Figs. (6.6a, b, and c), respectively. In each of these figures, $E_{1}$ and $E_{3}$ are eigenvalues of $H_{+}$, while $E_{2}$ and $E_{4}$ are eigenvalues of $H_{-}$.

In the first class of potentials (with barriers at both ends), equations similar to (6.15) govern both the left- and right-hand problems, generating plateaus near all the Dirichlet eigenvalues. Equations similar to (6.16) govern the interior wells, and generate transition regions. The apparent intersections of the solutions to the various equations are actually exponentially close near-crossings. Notice that there are eigenvalue trajectories near all the Dirichlet eigenvalues for all large values of $\lambda$.

The second class of potentials (with a barrier at one end) has already been discussed, in the specific example (H.6.3). In general, suppose that the exterior well is on the left and the exterior barriers is on the right (the other case is identical, 

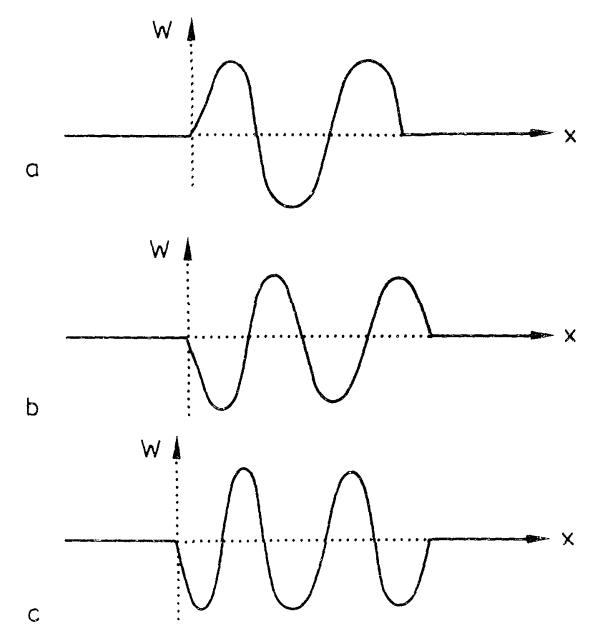

Fig. 6.5. Three classes of mixed-sign potentials

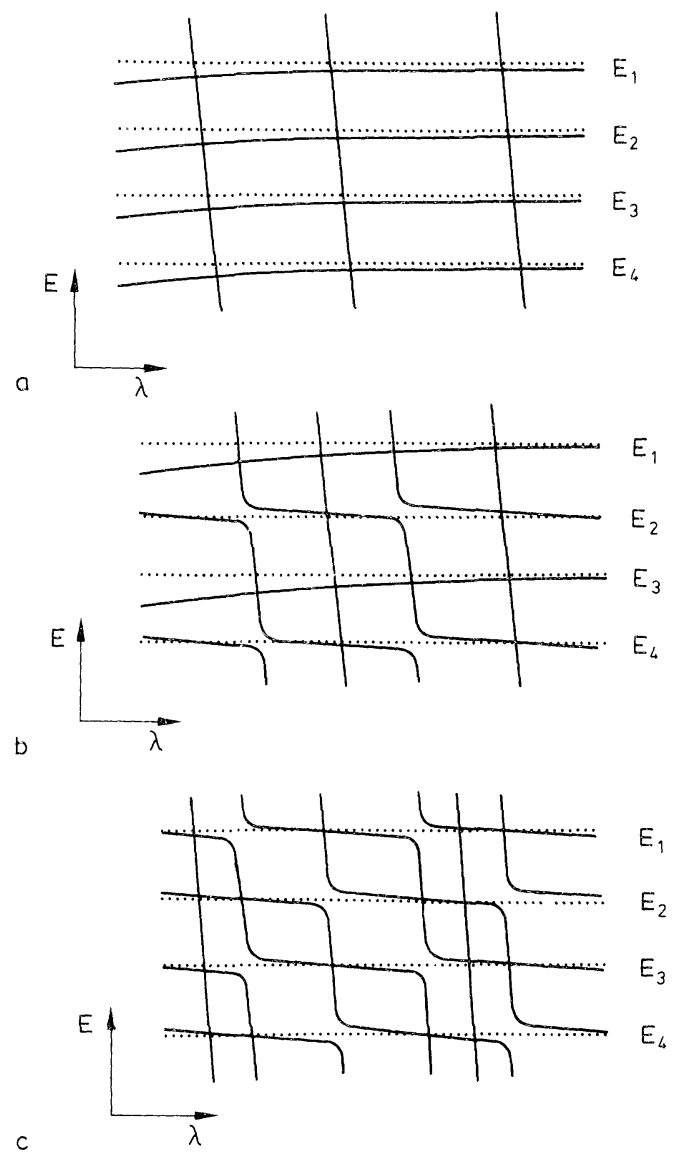

Fig. 6.6. Three classes of eigenvalue trajectories 
by symmetry). Then an equation similar to (6.12) governs the left-hand problem, generating both plateaus (at the eigenvalues of $H_{-}$) and transition regions. An equation similar to (6.15) governs the right-hand problem, and generates the plateaus near the eigenvalues of $H_{+}$. Equations similar to (6.16) govern the interior wells, and generate further transition regions. There are eigenvalues trajectories near the eigenvalues of $H_{+}$for all values of $\lambda$, but near the eigenvalues of $H_{-}$ there are some gaps.

For the third class of potentials (with wells at both ends), equations similar to (6.12) govern both the left- and right-hand problems, generating both plateaus and transition regions. The interior wells contribute additional transition regions via equations similar to $(6.16)$.

\section{Potentials of Point Support}

As mentioned in Remark 5.6, the cascade phenomenon depends on the support of $W$. In particular, if $W$ has point support the effect vanishes. For example, in a square well of width $\varepsilon$ and depth $1 / \varepsilon$ the period $L=\int \sqrt{|W|}=\varepsilon^{1 / 2}$. The first transition region occurs at $\lambda \approx \pi^{2} / L^{2}=\pi^{2} / \varepsilon$. Taking the $\delta$-function limit (i.e. $\varepsilon \rightarrow 0$ ), the first plateau extends to infinity, and the cascade phenomenon disappears.

The purpose of this section is to treat an analytically solvable model where $W$ has point support. Formally, this model has a background potential

$$
V(x)=\alpha \sum_{n \in \mathbf{Z}} \delta(x-a n)+\alpha \sum_{n \in \mathbf{Z}} \delta(x-a \rho-a n), \quad \alpha \in \mathbf{R} \backslash\{0\}, \quad \rho \in[0,1], \quad a>0,
$$

representing a diatomic Kronig-Penney model [27] and a perturbation

$$
W(x)=\lambda \delta(x), \quad \lambda \in \mathbf{R},
$$

describing a substitutional impurity. A precise definition of this model in $L^{2}(\mathbf{R})$ reads

$$
\begin{aligned}
H_{0}(\rho)= & -\frac{d^{2}}{d x^{2}} \\
\mathscr{D}\left(H_{0}(\rho)\right)= & \left\{g \in H^{1}(\mathbf{R}) \cap H^{2}(\mathbf{R} \backslash\{a \mathbf{Z} \cup a(\mathbf{Z}+\rho)\}) \mid g^{\prime}\left((n+\tau) a_{+}\right)-g^{\prime}\left((n+\tau) a_{-}\right)\right. \\
= & \left.\alpha\left(1+\delta_{0 \rho}+\delta_{1 \rho}\right) g((n+\tau) a), \tau \in\{0, \rho\}, n \in \mathbf{Z}\right\}, \\
& \alpha \in \mathbf{R} \backslash\{0\}, \quad \rho \in[0,1], \quad a>0
\end{aligned}
$$

and

$$
\begin{aligned}
H_{\lambda}(\rho)= & -\frac{d^{2}}{d x^{2}}, \\
\mathscr{D}\left(H_{\lambda}(\rho)\right)= & \left\{g \in H^{1}(\mathbf{R}) \cap H^{2}(\mathbf{R} \backslash\{a \mathbf{Z} \cup a(\mathbf{Z}+\rho)\}) \mid g^{\prime}\left((n+\tau) a_{+}\right)-g^{\prime}\left((n+\tau) a_{-}\right)\right. \\
= & {\left.\left[\alpha\left(1+\delta_{0 \rho}+\delta_{1 \rho}\right)+\lambda\left(\delta_{0 n} \delta_{0 \tau}+\delta_{-1 n} \delta_{1 \tau}\right)\right] g((n+\tau) a), \tau \in\{0, \rho\}, n \in \mathbf{Z}\right\}, } \\
& \alpha \in \mathbf{R} \backslash\{0\}, \quad \rho \in[0,1], \quad \lambda \in \mathbf{R}, a>0 .
\end{aligned}
$$

We also introduce in $L^{2}(\mathbf{R})$

$$
H_{\infty}(\rho)=-\frac{d^{2}}{d x^{2}},
$$




$$
\begin{aligned}
\mathscr{D}\left(H_{\infty}(\rho)\right) & =\left\{g \in H^{1}(\mathbf{R} \backslash\{0\}) \cap H^{2}(\mathbf{R} \backslash\{a \mathbf{Z} \cup a(\mathbf{Z}+\rho)\}) \mid g\left(0_{ \pm}\right)=0,\right. \\
g^{\prime}\left((n+\tau) a_{+}\right)-g^{\prime}\left((n+\tau) a_{-}\right) & =\alpha\left(1+\delta_{0 \rho}+\delta_{1 \rho}\right) g((n+\tau) a), \tau \in\{0, \rho\}, n \in \mathbf{Z} \backslash\left\{-\delta_{1 \tau}\right\}, \\
a & \in \mathbf{R} \backslash\{0\}, \quad \rho \in[0,1], \quad a>0
\end{aligned}
$$

and obtain the obvious decompsition

$$
H_{\infty}(\rho)=H_{-}(\rho) \oplus H_{+}(\rho), \quad \rho \in[0,1],
$$

where $H_{ \pm}(\rho)$ act in $L^{2}((0, \pm \infty))$. We first state

Lemma 7.1. Let $\alpha \in \mathbf{R} \backslash\{0\}$.

(i)

$$
\begin{array}{cc}
\sigma\left(H_{0}(\rho)\right)=\sigma\left(H_{0}(1-\rho)\right), & \rho \in[0,1], \\
\sigma\left(H_{+}(\rho)\right)=\sigma\left(H_{-}(1-\rho)\right), & \rho \in[0,1] .
\end{array}
$$

(ii) For all $\lambda \in \mathbf{R}, H_{\lambda}(\rho)$ has at most one eigenvalue in any spectral gap of $H_{0}(\rho)$, $\rho \in[0,1]$.

(iii)

$$
\sigma\left(H_{\infty}(\rho)\right)=\sigma\left(H_{0}(\rho)\right) \quad \text { for } \quad \rho=0,1 \text {. }
$$

In particular,

$$
\sigma_{p}\left(H_{\infty}(\rho)\right)=\emptyset \quad \text { for } \quad \rho=0, \frac{1}{2}, 1 .
$$

$$
\begin{gathered}
n-\lim _{i \rightarrow \pm \infty}\left(H_{\lambda}(\rho)-z\right)^{-1}=\left(H_{\infty}(\rho)-z\right)^{-1}, \quad \rho \in[0,1], \quad z \in \mathbf{C} \backslash \sigma\left(H_{\infty}(\rho)\right) . \\
\sigma_{\text {ess }}\left(H_{0}(\rho)\right)=\sigma_{\text {ess }}\left(H_{\lambda}(\rho)\right)=\sigma_{\text {ess }}\left(H_{\infty}(\rho)\right), \quad \rho \in[0,1], \quad \lambda \in \mathbf{R},
\end{gathered}
$$

and all singular continuous spectra are empty.

Proof. Let $P$ denote the unitary parity operator $(P g)(x)=g(-x), g \in L^{2}(\mathbf{R})$ or $g \in L^{2}((0, \infty))$, then

$$
P H_{0}(\rho) P^{-1}=H_{0}(1-\rho), \quad P H_{+}(\rho) P^{-1}=H_{-}(\rho)
$$

proves (i). Let $R_{\lambda}\left(\rho, z, x, x^{\prime}\right), R_{0}\left(\rho, z, x, x^{\prime}\right)$ and $R_{\infty}\left(\rho, z, x, x^{\prime}\right)$ denote the integral kernels of $\left(H_{\lambda}(\rho)-z\right)^{-1},\left(H_{0}(\rho)-z\right)^{-1}$, and $\left(H_{\infty}(\rho)-z\right)^{-1}, z \in \mathbf{C} \backslash \mathbf{R}$ respectively. Then

$$
\begin{aligned}
R_{\lambda}\left(\rho, z, x, x^{\prime}\right) & =R_{0}\left(\rho, z, x, x^{\prime}\right)-\frac{\lambda R_{0}(\rho, z, x, 0) R_{0}\left(\rho, z, 0, x^{\prime}\right)}{\lambda R_{0}(\rho, z, 0,0)+1}, \\
z & \in \mathbf{C} \backslash\left(\sigma\left(H_{\lambda}(\rho)\right) \cup \sigma\left(H_{0}(\rho)\right)\right), \quad \rho \in[0,1], \quad \lambda \in \mathbf{R}, \quad x, x^{\prime} \in \mathbf{R}
\end{aligned}
$$

proves (ii). Taking the limit $\lambda \rightarrow \pm \infty$ in (7.14), observing (3.16), proves (iv). Relation (iii) follows from reflection symmetry with respect to $x=0$ as discussed after (3.18). Clearly (7.14) also proves (v).

Next we briefly describe the spectrum of $H_{0}(\rho)$.

Lemma 7.2. Let $\alpha \in \mathbf{R} \backslash\{0\}, \rho \in[0,1]$. Then $H_{0}(\rho)$ has purely absolutely continuous spectrum

$$
\sigma\left(H_{0}(\rho)\right)=\sigma_{\mathrm{ac}}\left(H_{0}(\rho)\right)=\bigcup_{m=1}^{\infty}\left[a_{m}(\rho), b_{m}(\rho)\right], \quad a_{m}(\rho)<b_{m}(\rho) \leqq a_{m+1}(\rho), \quad m \in \mathbf{N} .
$$


The discriminant associated with $H_{0}(\rho)$ reads

$$
\cos (\theta a)=\cos (k a)+\frac{\alpha}{k} \sin (k a)+\frac{\alpha^{2}}{2 k^{2}} \sin (k a \rho) \sin [k a(1-\rho)], \quad \theta \in[-\pi / a, \pi / a] .
$$

Let $k_{m}(\theta, \rho)$ be the solutions of (7.16) and introduce $E_{m}(\theta, \rho)=\left[k_{m}(\theta, \rho)\right]^{2}, m \in \mathbf{N}$ (ordered in magnitude). Then

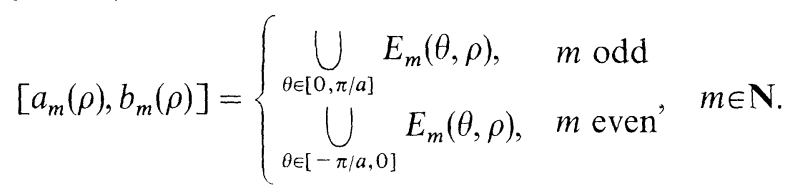

For all $\rho \in[0,1], H_{0}(\rho)$ has infinitely many open gaps (unless $\exists n \in \mathbb{N}$ s.t. $\cot [n \pi \rho /$ $(1-2 \rho)]=-\alpha a(1-2 \rho) / 2 n \pi$, all possible gaps are open; whereas for $\rho=1 / 2$ every second gap closes).

Proof. An operator similar to $H_{0}(\rho)$ has been analyzed in [27] using Green's function methods and constructing the Floquet solutions explicitly. Here we sketch an alternative possibility based on finite difference methods (see [3] and the references therein). The (generalized) eigenvalue equation

$$
H_{0}(\rho) \psi(k)=k^{2} \psi(k), \quad k^{2} \in \mathbf{R}, \quad \operatorname{Im} k \geqq 0
$$

is equivalent to

where

$$
M N \Phi_{j}=\Phi_{j+1}, \quad N M \Psi_{j}=\Psi_{j+1}, \quad j \in \mathbf{Z},
$$

$$
\begin{gathered}
M(k, \rho)=\left[\begin{array}{cc}
\frac{\alpha \sin (k a \rho)}{k}+\frac{\sin (k a)}{\sin [k a(1-\rho)]} & -\frac{\sin (k a \rho)}{\sin [k a(1-\rho)]} \\
1 & 0
\end{array}\right], \\
N(k, \rho)=\left[\begin{array}{cc}
\frac{\alpha \sin [k a(1-\rho)]}{k}+\frac{\sin (k a)}{\sin (k a \rho)} & -\frac{\sin [k a(1-\rho)]}{\sin (k a \rho)} \\
1 & 0
\end{array}\right]
\end{gathered}
$$

and

$$
\Phi_{j}(k)=\left[\begin{array}{c}
\psi((j+\rho) a) \\
\psi((j-1+\rho) a)
\end{array}\right], \quad \Psi_{j}(k)=\left[\begin{array}{c}
\psi(j a) \\
\psi((j-1) a)
\end{array}\right], \quad j \in \mathbf{Z}
$$

By Floquet's theorem, $\Phi_{j+1}=e^{i \theta a} \Phi_{j}$. Thus

$$
\operatorname{Tr}[M N]=2 \cos (\theta a),
$$

which is equivalent to (7.16). The rest of the assertions are standard (cf. e.g. [26]). Next we describe the point spectrum of $H_{\infty}(\rho)$.

Lemma 7.3. Let $\alpha \in \mathbf{R} \backslash\{0\}, \rho \in(0,1) \backslash\{1 / 2\}, m \in \mathbb{N}, m \rho \notin \mathbb{N}$. Then $H_{\infty}(\rho)$ has precisely one simple eigenvalue in the m-th spectral gap of $H_{0}(\rho) . H_{\infty}(\rho)$ has no eigenvalues in 
the zeroth gap (i.e. the gap starting at $-\infty$ ). The corresponding eigenvalue equation for these eigenvalues $E_{\infty}(\rho)=k^{2}$ reads

$$
k \sin (k a)+\alpha \sin (k a \rho) \sin [k a(1-\rho)]=0, \quad \operatorname{Im} k \geqq 0 .
$$

$H_{\infty}(\rho)$ has no other eigenvalues. More precisely,

$$
\sigma_{p}\left(H_{\infty}(\rho)\right)=\sigma_{d}\left(H_{\infty}(\rho)\right)=\sigma_{d}\left(H_{-}(\rho)\right) \cup \sigma_{d}\left(H_{+}(\rho)\right) .
$$

(For $\rho=0,1 / 2,1$ cf. Lemma 7.1).

Proof. Because of (7.14), all eigenvalues of $H_{\infty}(\rho)$ are simple and discrete. They are given by the solutions $E \in \mathbf{R} \backslash \sigma\left(H_{0}(\rho)\right)$ of

$$
R_{0}(\rho, E, 0,0)=0 \text {. }
$$

Moreover, because of (7.6), an eigenvalue of $H_{\infty}(\rho)$ is either an eigenvalue of $H_{-}(\rho)$ or of $H_{+}(\rho)$, but never of both simultaneously, since this would contradict its simplicity. By (7.8), it suffices to study $H_{+}(\rho)$. In principle we can study (7.25) directly, but we prefer to use again finite difference methods. The eigenvalue equation

$$
H_{+}(\rho) \psi_{+}(k)=k^{2} \psi_{+}(k), \quad \psi_{+}(k) \in \mathscr{D}\left(H_{+}(\rho)\right), \quad k^{2} \in \mathbf{R} \backslash \sigma\left(H_{0}(\rho)\right), \quad \operatorname{Im} k \geqq 0
$$

is equivalent to the coupled difference equations

$$
\begin{aligned}
& \sin [k a(1-\rho)] \psi_{j+\rho}(k)+\sin (k a \rho) \psi_{j-1+\rho}(k)=A(\rho, k) \psi_{j}(k), \\
& \sin (k a \rho) \psi_{j+1}(k)+\sin [k a(1-\rho)] \psi_{j}(k)=A(\rho, k) \psi_{j+\rho}(k), \\
& \psi_{j+\tau}(k):=\psi_{+}(k,(j+\tau) a), \quad \tau \in\{0, \rho\}, \quad j \in \mathbf{N}_{0}, \quad\left\{\psi_{j}(k), \psi_{j+\rho}(k)\right\}_{j \in \mathbf{N}_{0}} \in l^{2}\left(\mathbf{N}_{0}\right),
\end{aligned}
$$

where

$$
A(\rho, k)=\sin (k a)+\frac{\alpha}{k} \sin (k a \rho) \sin [k a(1-\rho)] .
$$

A careful analysis of (7.26) reveals the fact that $\left\{\psi_{j}(k), \psi_{j+\rho}(k)\right\}_{j \in \mathbf{N}_{0}} \notin l^{2}\left(\mathbf{N}_{0}\right)$, and hence $\psi_{+}(k) \notin L^{2}((0, \infty))$ unless $A(\rho, k)=0$, i.e. unless (7.23) holds. Setting the right-hand side of (7.27) equal to zero then yields

$$
\psi_{j}(k)=0, \quad \psi_{j+\rho}(k)=\frac{(-1)^{j} \sin ^{j+1}(k a \rho)}{\sin ^{j}[k a(1-\rho)]}, \quad j \in \mathbf{N}_{0}
$$

Thus, defining $y(\rho, k):=\sin (k a \rho) / \sin [k a(1-\rho)]$, we have

$$
\left\{\psi_{j+\rho}(k)\right\}_{\jmath \in \mathbf{N}_{0}} \in l^{2}\left(\mathbf{N}_{0}\right) \text { iff }|y(\rho, k)|<1 .
$$

Similarly

$$
\begin{gathered}
H_{-}(\rho) \psi_{-}(k)=k^{2} \psi_{-}(k), \quad \psi_{-}(k) \in \mathscr{D}\left(H_{-}(\rho)\right), \quad k^{2} \in \mathbb{R} \backslash \sigma\left(H_{0}(\rho)\right), \\
\operatorname{Im} k \geqq 0 \quad \text { iff } \quad|y(\rho, k)|>1 .
\end{gathered}
$$

Given all these results we can finally describe the point spectrum of $H_{\lambda}(\rho)$. 
Theorem 7.4. Let $\alpha, \lambda \in \mathbf{R} \backslash\{0\}$ and $\rho \in[0,1]$. Then $H_{\lambda}(\rho)$ has precisely one simple eigenvalue in the zeroth gap if $\lambda<0$ and no eigenvalue in the zeroth gap if $\lambda>0$. For $m \in \mathbb{N}, m \rho \notin \mathbb{N}, \rho \neq 0, H_{\lambda}(\rho)$ has precisely one simple eigenvalue in the $m$-th spectral gap of $H_{0}(\rho)$. The corresponding eigenvalue equation for these eigenvalues $E_{\lambda}(\rho)=k^{2}$ reads

$$
\begin{aligned}
& \left\{\sin (k a)+\frac{\alpha}{k} \sin (k a \rho) \sin [k a(1-\rho)]\right\}^{2} \\
& \quad=\frac{4 k^{2}}{\lambda^{2}}\left\{\cos (k a)+\frac{\alpha^{2}}{2 k^{2}} \sin (k a \rho) \sin [k a(1-\rho)]+\frac{\alpha}{k} \sin (k a)\right\}^{2}-\frac{4 k^{2}}{\lambda^{2}}, \quad \operatorname{Im} k \geqq 0 .
\end{aligned}
$$

Each eigenvalue $E_{\lambda}(\rho)$ of $H_{\lambda}(\rho), \rho \in(0,1) \backslash\{1 / 2\}, m \in \mathbb{N}, \rho m \notin \mathbb{N}$ converges to the corresponding eigenvalue $E_{\infty}(\rho)$ of $H_{\infty}(\rho)$ in the $m$-th spectral gap of $H_{0}(\rho)$ as $\lambda \rightarrow \pm \infty$ (from below for $\lambda \rightarrow \infty$ and from above as $\lambda \rightarrow-\infty$ ), with one exception: the ground state of $H_{\lambda}(\rho)$ converges to $-\infty$ as $\lambda \rightarrow-\infty$. For $\rho=0,1 / 2,1$ all eigenvalues of $H_{\lambda}(\rho)$ are absorbed into the (absolutely continuous) spectrum of $H_{0}(\rho)$ as $\lambda \rightarrow \pm \infty$, except that the ground state of $H_{\lambda}(\rho)$ again converges to $-\infty$ as $\lambda \rightarrow-\infty$. For $m \rho \in \mathbb{N}, \rho \neq 0, \frac{1}{2}, 1, H_{\lambda}(\rho)$ has no eigenvalues in the $m$-th spectral gap of $H_{0}(\rho)$.

Proof. Because of (7.10) and (7.11) it only remains to derive (7.32). Using again the difference scheme one arrives at

$$
\begin{aligned}
& \cos (\theta a)=\cos (k a)+\frac{\alpha^{2}}{2 k^{2}} \sin (k a \rho) \sin [k a(1-\rho)]+\frac{\alpha}{k} \sin (k a), \\
& \sin (\theta a)=\frac{\lambda}{2 k i}\left\{\sin (k a)+\frac{\alpha}{k} \sin (k a \rho) \sin [k a(1-\rho)]\right\} .
\end{aligned}
$$

Remark 7.5. Theorem 7.4 thus serves as a "counterexample" to Sect. 5 in that, in contrast to the cascade phenomenon, there exists a norm resolvent limit to a Dirichlet operator $H_{\infty}(\rho)$ as $\lambda \rightarrow \pm \infty$, and a corresponding convergence of the spectrum. However, since $\operatorname{supp}(W)=\{0\}$ in this special case, $\lambda W$ can at most support one additional bound state in each spectral gap of $H_{0}(\rho)$ for all $\lambda \in \mathbf{R}$. As a consequence, this additional eigenvalue simply converges to the (unique) Dirichlet level of $H_{\infty}(\rho)$ in any spectral gap of $H_{0}(\rho)$ (except the zeroth one).

Remark 7.6. The basic equation (7.14) actually holds for any real-valued background potential $V \in L^{\infty}(\mathbf{R})$ as long as we keep $\lambda W(x)=\lambda \delta\left(x-x_{0}\right)$ of point support. In obvious notation (cf. Sect. 3) one gets

and

$$
\begin{aligned}
R_{\lambda}\left(z, x, x^{\prime}\right) & =R_{0}\left(z, x, x^{\prime}\right)-\frac{\lambda R_{0}\left(z, x, x_{0}\right) R_{0}\left(z, x_{0}, x^{\prime}\right)}{\lambda R_{0}\left(z, x_{0}, x_{0}\right)+1}, \\
z & \in \mathbf{C} \backslash\left(\sigma\left(H_{\lambda}\right) \cup \sigma\left(H_{0}\right)\right), \quad \lambda \in \mathbf{R}, \quad x, x^{\prime} \in \mathbf{R}
\end{aligned}
$$

$$
\begin{aligned}
R_{\infty}\left(z, x, x^{\prime}\right) & =R_{0}\left(z, x, x^{\prime}\right)-\frac{R_{0}\left(z, x, x_{0}\right) R_{0}\left(z, x_{0}, x^{\prime}\right)}{R_{0}\left(z, x_{0}, x_{0}\right)}, \\
z & \in \mathbf{C} \backslash\left(\sigma\left(H_{\infty}\right) \cup \sigma\left(H_{0}\right)\right), \quad x, x^{\prime} \in \mathbf{R} .
\end{aligned}
$$


In particular this implies

$$
\lim _{\lambda \rightarrow \pm \infty}\left(H_{\lambda}-z\right)^{-1}=\left(H_{\infty}-z\right)^{-1}, \quad z \in \mathbf{C} \backslash \sigma\left(H_{\infty}\right),
$$

and hence convergence of the point spectrum (i.e. no cascade phenomenon).

Remark 7.7. One could easily repeat this section for other potentials of point support (e.g. for the analog of $\delta^{\prime}\left(x-x_{0}\right)$-potentials discussed in great detail in [3]).

\section{Eigenvalue Trapping for Dirac Operators}

In this section we consider Dirac operators on the half-line $\mathbf{R}_{+}:=(0, \infty)$, in the large-coupling limit. In contrast to the Schrödinger case discussed before, we do not see the cascade phenomenon. Instead, as $\lambda \rightarrow \infty$, the eigenvalues $E_{\lambda}$ of the Dirac Hamiltonian $H_{\lambda}$ either approach an asymptotic value or do not converge, depending on whether a particular integral is zero or not.

Our hypotheses are:

(H.8.1) Assume $V \in C^{1}\left(\mathbf{R}_{+}\right)$to be real-valued with $\left|V\left(0_{+}\right)\right|<\infty$ and $V(r)=o(1)$ as $r \rightarrow \infty$.

For square-well-like potentials we have

(H.8.2) Let $W$ be real-valued with support $[0, l], l>0$ and $C^{1}$ except at a finite number of points $\left\{0, p_{1}, \ldots, p_{n}, l\right\} \subset[0, l]$, where it may have a jump discontinuity. Moreover, we assume $|W(r)| \geqq \varepsilon>0$ for all $r \in(0, l)$.

For turning-point potentials we introduce

$W(r)=\left\{\begin{array}{ll}0 & r>l, \\ \tilde{W}(r) & r \leqq l,\end{array} \quad l>0, \quad\right.$ where $\tilde{W}(r)$ is $C^{1}$ and real-valued with $\left|\tilde{W}\left(0_{+}\right)\right|<\infty$.

In addition we suppose that $\tilde{W}$ has a finite number of zeroes, including one at $r=l$, all of which are simple.

Given (H.8.1) and either (H.8.2) or (H.8.3) we define the Dirac Hamiltonian $H_{\lambda}$ in $L^{2}\left(\mathbf{R}_{+}\right)^{2}$ to be

$$
\begin{gathered}
H_{\lambda}=\left(\begin{array}{rr}
0 & -1 \\
1 & 0
\end{array}\right) \frac{d}{d r}+\left(\begin{array}{cc}
1+\lambda W & V \\
V & -1+\lambda W
\end{array}\right), \\
\mathscr{D}\left(H_{\lambda}\right)=\left\{g=\left(\begin{array}{l}
g_{1} \\
g_{2}
\end{array}\right) \in H^{1}\left(\mathbf{R}_{+}\right)^{2} \mid g_{1}\left(0_{+}\right)=0\right\}, \quad \lambda \geqq 0 .
\end{gathered}
$$

For later purposes we also remark that for real-valued, locally absolutely continuous solutions $\Psi$,

$$
\left(\begin{array}{rr}
0 & -1 \\
1 & 0
\end{array}\right) \Psi^{\prime}(E, r)+\left(\begin{array}{cc}
1+\lambda W-E & V \\
V & -1+\lambda W-E
\end{array}\right) \Psi(E, r)=0, \quad E \in \mathbf{R}, \quad r>0
$$


is equivalent to

$$
\begin{aligned}
& \theta^{\prime}(E, r)=E-\lambda W(r)+\cos [2 \theta(E, r)]-V(r) \sin [2 \theta(E, r)], \\
& \rho^{\prime}(E, r)=\rho(E, r)\{\sin [2 \theta(E, r)]+V(r) \cos [2 \theta(E, r)]\}, \quad E \in \mathbf{R}, \quad r>0,
\end{aligned}
$$

where (cf. the Prüfer transformation in [34])

$$
\begin{aligned}
& \psi_{1}(E, r)=\rho(E, r) \sin [\theta(e, r)] \\
& \psi_{2}(E, r)=\rho(E, r) \cos [\theta(e, r)], \quad E \in \mathbf{R}, \quad r>0 .
\end{aligned}
$$

Remark 8.1. Hypotheses (H.8.1) and either (H.8.2) or (H.8.3) ensure that $\sigma_{\text {ess }}\left(H_{\lambda}\right)=$ $(-\infty,-1] \cup[1, \infty), \lambda \geqq 0[34]$. They also guarantee that $H_{0}$ is in the limit point case at $\infty[34]$. Thus, given $E \in(-1,1)$ we can find a real-valued $\mathbf{f}$ such that

$$
H_{0} \mathbf{f}=E \mathbf{f} \text { (distributional sense), } \mathbf{f}=\left(\begin{array}{c}
f_{1} \\
f_{2}
\end{array}\right) \in H^{1}\left(\mathbf{R}_{+}\right)^{2}
$$

We take, in analogy to (8.4),

$$
\begin{aligned}
& f_{1}(E, r)=\rho_{0}(E, r) \sin \left[\theta_{0}(E, r)\right] \\
& f_{2}(E, r)=\rho_{0}(E, r) \cos \left[\theta_{0}(E, r)\right],
\end{aligned} \quad E \in(-1,1), \quad r>0 .
$$

Then

$$
\theta_{0}\left(-1_{+}, r\right)=0, \quad r>0,
$$

and $\theta_{0}(E, r), r>0$ is monotonically decreasing with respect to $E \in(-1,1)$. (To see this, note that (8.3) implies that $\theta_{0}(E, \infty)=-\frac{1}{2} \cos ^{-1}(-E)$, a decreasing function of $E$. Thus if we let $-1<E_{1}<E_{2}<1$, we have $\theta_{0}\left(E_{1}, r\right)>\theta_{0}\left(E_{2}, r\right)$ for all $r>0$ sufficiently large. Now suppose that $\theta_{0}\left(E_{1}, r_{0}\right)=\theta_{0}\left(E_{2}, r_{0}\right)$ for some $r_{0}$ and that $\theta_{0}\left(E_{1}, r\right)>\theta_{0}\left(E_{2}, r\right)$ for all $r>r_{0}$. However, (8.3a) states that $\theta^{\prime}\left(E_{2}, r_{0}\right)-\theta^{\prime}\left(E_{1}, r_{0}\right)=$ $E_{2}-E_{1}>0$, so $\theta_{0}\left(E_{2}, r\right)>\theta_{0}\left(E_{1}, r\right)$ for $r \in\left(r_{0}, r_{0}+\varepsilon\right)$ for some $\varepsilon>0$. This contradiction shows that $r_{0}$ does not exist, and so $\theta_{0}(E, r)$ is monotonically decreasing with respect to $E \in(-1,1)$.)

Our main result then reads

Theorem 8.2. Assume (H.8.1) and either (H.8.2) or (H.8.3).

(i) If $\int_{0}^{l} W(r) d r=0$, then, as $\lambda \rightarrow \infty$, the eigenvalues $E_{\lambda}$ of $H_{\lambda}$ converge to solution(s) $E_{\infty}$ of the equation

$$
E l \equiv \theta_{0}(E, l) \quad(\bmod \pi)
$$

if such solutions exist. In this case $E_{\lambda}-E_{\infty}=O\left(\lambda^{-\alpha}\right)$, where $\alpha=1$ if (H.8.2) holds and $\alpha=1 / 2$ if (H.8.3) holds.

(ii) If $\int_{0}^{l} W(r) d r \neq 0$, then $E_{\lambda}$ does not converge as $\lambda \rightarrow \infty$. Rather, $E_{\lambda}$ satisfies

$$
E_{\lambda} l-\lambda \int_{0}^{l} W(r) d r+O\left(\lambda^{-\alpha}\right) \equiv \theta_{0}\left(E_{\lambda}, l\right) \quad(\bmod \pi)
$$

as $\lambda \rightarrow \infty$. 
Proof. We consider solutions $\Psi$ of $H_{\lambda} \Psi=E \Psi$ on $(0, l)$ and match them to the exterior solutions $\mathbf{f}$ of $H_{0} \mathbf{f}=E \mathbf{f}$ (cf. Remark 8.1) at the point $l$. Then (8.3) implies

$$
\begin{aligned}
\theta(E, r) & =E r-\lambda \int_{0}^{r} W(s) d s+\int_{0}^{r} \cos [2 \theta(E, s)] d s-2 \int_{0}^{r} V(s) \sin [2 \theta(E, s)] d s, \\
\theta_{0}\left(E, 0_{+}\right) & =0, \quad E \in(-1,1), \quad 0<r \leqq l .
\end{aligned}
$$

Iterating (8.10) once, then taking $r=l$, it is not difficult to show that

$$
\theta(E, l)-E l+\lambda \int_{0}^{l} d s W(s)=O\left(\lambda^{-\alpha}\right)
$$

as $\lambda \rightarrow \infty$ with $\alpha$ as above. In the square-well-like case (H.8.2), one need only integrate by parts dividing $(0, l)$ into $\left(0, p_{1}\right) \cup\left(p_{1}, p_{2}\right) \cup \cdots \cup\left(p_{n}, l\right)$. In the turningpoint case (H.8.3), one must also employ a truncation technique at the turning points. Setting $\theta(E, l) \equiv \theta_{0}(E, l)(\bmod \pi)$ then establishes $(8.9)$. Since $\theta_{0}(E, l)$ is a decreasing function with respect to $E \in(-1,1)$ by Remarks 8.1, the $O\left(\lambda^{-\alpha}\right)$ errors in the phase (8.9) give rise to $O\left(\lambda^{-\alpha}\right)$ errors in the energy. This proves (8.8) if $\int_{0}^{l} d r W(r)=0$.

Remark 8.3. Theorem 8.2 could be extended to $W$ 's that have mutliple zeroes. In fact, if $W$ has $n$-th order zeroes, then $\alpha$ becomes $(n+1)^{-1}$.

Remark 8.4. In the free case $(V(r)=0$ for $r>l)$ one has

$$
\theta_{0}(E, l)=-\tan ^{-1}\left(\sqrt{\frac{1+E}{1-E}}\right), \quad E \in(-1,1)
$$

whereas in the Coulomb case $(V(r)=-1 / r$ for $r>l)$ one has

$$
\theta_{0}(E, l)=-\tan ^{-1}\left(\frac{1+E}{\sqrt{1-E^{2}+l^{-1}}}\right) \quad E \in(-1,1) .
$$

In both cases (8.8) always has a solution (even for arbitrarily small $l>0$ ).

Remark 8.5. As can be read off from Theorem 8.2, $H_{\lambda}$ does not converge to a limiting operator $H_{\infty}$ as $\lambda \rightarrow \infty$. Instead, if $\int_{0}^{l} d r W(r)=0, E_{\lambda}$ converges to the solution(s) of the eigenvalue problem $\left(\begin{array}{rr}0 & -1 \\ 1 & 0\end{array}\right) \mathbf{f}^{\prime}+\left(\begin{array}{cc}1-E & V \\ V & -1-E\end{array}\right) \mathbf{f}=0$ in $L^{2}((l, \infty))^{2}$ with the energy dependent boundary condition $E l \equiv \theta_{0}\left(E, l_{+}\right)(\bmod \pi)$.

Acknowledgements. We thank Anita Glazer for her draftsmanship. F. Gesztesy and D. Gurarie are indebted to D. Wales for the hospitality extended to them at Caltech. F. Gesztesy gratefully acknowledges support from the Max Kade Foundation and from USNSF under Grant DMS-8416049. H. Holden appreciates discussions with G. Taraldsen. 


\section{References}

1. Abramowitz, M., Stegun, I. A.: Handbook of mathematical functions. New York: Dover 1972

2. Alama, S., Deift, P., Hempel, R.: Eigenvalue problems arising in the theory of the color of crystals (to appear)

3. Albeverio, S., Gesztesy, F., Hфegh-Krohn, R., Holden, H.: Solvable models in quantum mechanics. Texts and Monographs in Physics. Berlin, Heidelberg, New York: Springer 1988

4. Ashbaugh, M.S., Harrell, E. M.: Perturbation theory for shape resonances and large barrier potentials. Commun. Math. Phys. 83, 151-170 (1982)

5. Baumgärtel, H., Demuth, M.: Decoupling by a projection, Rep. Math. Phys. 15, 173-186 (1979)

6. Bulla, W., Gesztesy, F.: Deficiency indices and singular boundary conditions in quantum mechanics. J. Math. Phys. 26, 2520-2528 (1985)

7. Deift, P. A.: Applications of a communication formula, Duke Math. J. 45, 267-310 (1978)

8. Deift, P. A., Hempel, R.: On the existence of eigenvalues of the Schrödinger operator $H-\lambda W$ in a gap of $\sigma(H)$. Commun. Math. Phys. 103, 461-490 (1986)

9. Dunford, N., Schwartz, J. T.: Linear Operators II. New York: Interscience 1963

10. Gesztesy, F., Simon, B.: On a theorem of Deift and Hempel. Commun. Math. Phys. 116, 503-505 (1988)

11. Grinberg, A. A.: Energy spectrum of an electron placed in the fields of a small-radius potential well and an attractive coulomb potential. Sov. Phys. Semicond. 11, 1118-1120 (1977)

12. Harrell, E. M.: The band structure of a one-dimensional periodic system in a scaling limit. Ann. Phys. 119, 351--369 (1979)

13. Hempel, R.: A left-indefinite generalized eigenvalue problem for Schrödinger operators. Habilitationsschrift, University of Munich, FRG, 1987

14. Hempel, R., Hinz, A. M., Kalf, H.: On the essential spectrum of Schrödinger operators with spherically symmetric potentials. Math. Ann. 277, 197-208 (1987)

15. Hille, E., Phillips, R. S.: Functional analysis and semigroups. Rev. ed., Providence, RI: Am. Math. Soc. Colloq. Publ. 31, (1957)

16. Kato, T.: On the Trotter-Lie product formula. Proc. Jpn. Acad. 50, 694-698 (1974)

17. Kato, T.: Trotter's product formula for an arbitrary pair of self-adjoint contraction semi-groups. In: Topics in functional analysis. Adv. Math. Suppl. Stud. 3, 185-195 (1978)

18. Kato, T.: Monotonicity theorems in scattering theory. Hadronic J. 1, 134-154 (1978)

19. Kato, T.: Perturbation theory for linear operators. 2nd corr. (ed.), Berlin, Heidelberg, New York: Springer 1980

20. Klaus, M.: Some applications of the Birman-Schwinger principle. Helv. Phys. Acta 55, 49-68 (1982)

21. Kudryavtsev, A. E., Markushin, V. E., Shapiro, I. S.: Nuclear level shift in the ( $p \bar{p})$ Atom. Sov. Phys. JETP 47, 225-232 (1978)

22. Olver, F. W. J.: Asymptotics and special functions. New York: Academic Press 1974

23. Pötz, W., Vogl, P.: High magnetic field effects on shallow and deep impurities in semiconductors. Solid State Comm. 48, 249-252 (1983)

24. Popov, V. S.: On the properties of the discrete spectrum for $Z$ close to 137. Sov. Phys. JETP 33, 665-673 (1971)

25. Reed, M., Simon, B.: Methods of modern mathematical physics I: Functional analysis, rev. and enlarged ed., New York: Academic Press 1980

26. Reed, M., Simon, B.: Methods of modern mathematical physics IV: Analysis of operators. New York: Academic Press 1978

27. Saxon, D. S., Hutner, R. A.: Some electronic properties of a one-dimensional crystal model. Phillips Res. Rep. 4, 81-122 (1949)

28. Simon, B.: Coupling constant analyticity for the anharmonic oscillator. Ann. Phys. 58, 76-136 (1970)

29. Simon, B.: Lower semicontinuity of positive quadratic forms. Proc. Roy. Soc. Edinburgh 79, 267-273 (1977)

30. Simon, B.: A canonical decomposition for quadratic forms with applications to monotone convergence theorems. J. Funct. Anal. 28, 377-385 (1978) 
31. Thirring, W.: A course in mathematical physics Vol. 3: Quantum mechanics of atoms and molecules. Berlin, Heidelberg, New York: Springer 1981

32. Veselić, K.: Perturbation of pseudoresolvents and analyticity in $1 / c$ in relativistic quantum mechanics. Commun. Math. Phys. 22, 27-43 (1971)

33. Vogl, P.: Chemical trends of deep impurity levels in covalent semiconductors. In: Festkörperprobleme XXI (1981), pp. 191-219. Wiesbaden, Vieweg

34. Weidmann, J.: Oszillationsmethoden für systeme Gewöhnlicher Differentialgleichungen. Math. Z. 119, 349-373 (1971)

35. Weidmann, J.: Linear operators in Hilbert spaces. Graduate texts in mathematics Vol. 68, Berlin, Heidelberg, New York: Springer 1980

36. Zel'dovich, Ya.B.: Energy levels in a distorted Coulomb field. Sov. Phys. Solid State 1, 1497-1501 (1960)

Communicated by A. Jaffe

Received February 29, 1988 